\title{
East Texas Caddo Ceramic Sherd Database
}

Timothy K. Perttula

Heritage Research Center, Stephen F. Austin State University

Follow this and additional works at: https://scholarworks.sfasu.edu/ita

Part of the American Material Culture Commons, Archaeological Anthropology Commons, Environmental Studies Commons, Other American Studies Commons, Other Arts and Humanities Commons, Other History of Art, Architecture, and Archaeology Commons, and the United States History Commons

Tell us how this article helped you.

This Article is brought to you for free and open access by the Center for Regional Heritage Research at SFA ScholarWorks. It has been accepted for inclusion in Index of Texas Archaeology: Open Access Gray Literature from the Lone Star State by an authorized editor of SFA ScholarWorks. For more information, please contact cdsscholarworks@sfasu.edu. 


\section{East Texas Caddo Ceramic Sherd Database}

Creative Commons License

(c) (i) (8)

This work is licensed under a Creative Commons Attribution-NonCommercial 4.0 International License 


\title{
East Texas Caddo Ceramic Sherd Database
}

\author{
Timothy K. Perttula
}

\section{INTRODUCTION}

A considerable amount of effort has been expended over the years by archaeologists in the identification, description, and classification of ancestral Caddo ceramic vessels and sherds recovered from sites across East Texas, beginning with the masterful efforts of Alex D. Krieger $(1941,1946)$. These analyses have led to an appreciation of the stylistic, technological, functional, and morphological character of Caddo ceramics, as well as their age, and their role in the identification and scale of social networks of different Caddo communities in existence as early as ca. A.D. 850 to the early 19 th century.

The purpose of the compilation of attribute-level data on Caddo ceramic sherds in East Texas is to build on the understandings already achieved through many years of study by numerous individuals regarding the stylistic, technological, and functional character of Caddo ceramics (see Perttula 2013i). This compilation is a distillation of 50+ years of the analysis and study of Caddo ceramics - particularly the quantification of the methods of decorations present on sherds from different assemblages - and a compilation that is useful for both present and future detailed studies of the sherds from ceramic vessel made by perhaps 40 or more generations of skilled Caddo potters.

\section{CERAMIC SHERD DATABASE}

The East Texas Caddo ceramic sherd database (Table 1) presents uniform information on the character of ceramic assemblages on East Texas Caddo sites of different ages and components within sites collected from published reports, articles, and manuscripts (Figure 1). Ceramic data on a uniform set of attributes has been gathered from these sources of information, even when information is not available on all the attributes in the assemblages. The database contains assemblage-level information from 399 Caddo sites and/ or components in East Texas and eight sites and/or components from sites along the Sabine River at Toledo Bend Reservoir in western Louisiana (Table 1). To date, the East Texas Caddo ceramic sherd database contains information on the occurrence and relative percentages of 248,148 decorated sherds from these sites/components, while there is similar information on 11,948 decorated sherds from the western Louisiana Caddo sites; the total number of decorated sherds in the database as of August 2014 is 260,096. For present purposes, the database entries were restricted to sites and assemblages with more than 40 decorated sherds in areas where decorated sherd assemblages were small (i.e., the upper Sulphur River drainage and upper Red River drainage), or with more than 90-100 sherds elsewhere in the region. On average, an individual site or assemblage in the database from East Texas contains ca. 620 decorated sherds, while the western Louisiana sites in the database contain ca. 1500 decorated sherds on average.

The database includes percentage information on the following attributes: (a) temper (i.e., grog, bone, shell, grog-bone, and sandy paste); (b) firing conditions (reduced, oxidized, incompletely oxidized, and reduced-oxidized); (c) rim profile (direct, inverted, and everted); (d) lip profile (rounded, flat, rounded-exterior folded, and beveled); (e) use of pigments (red and white); (f) decorative methods in utility wares (appliqued, appliqued-brushed, appliqued-incised, appliqued-punctated, brushed, brushed-incised, brushed-punctated, brushed-appliqued, incised, incised-punctated, neck banded, pinched, tool punctated, fingernail punctated, 
Table 1. East Texas Caddo ceramic sherd database.

\begin{tabular}{|c|}
\hline Trinomial \\
\hline $41 \mathrm{CP} 495$ \\
\hline 41CP72 \\
\hline 41RA13 \\
\hline 41CE421 \\
\hline 41CE423 \\
\hline 41RA13 \\
\hline 41CE426 \\
\hline 41CE429 \\
\hline 41NA223 \\
\hline 41CE324 \\
\hline 41AN87 \\
\hline 41CE309 \\
\hline 41HE337 \\
\hline 41CE354 \\
\hline 41RK191 \\
\hline 41RK197 \\
\hline 41RK200 \\
\hline $41 \mathrm{CP} 230$ \\
\hline 41WD52 \\
\hline 41FK107 \\
\hline 41SM195 \\
\hline 41TT650 \\
\hline 41UR279 \\
\hline $41 \mathrm{CP} 245$ \\
\hline $41 \mathrm{HS} 574$ \\
\hline 41BW171 \\
\hline 41WD46 \\
\hline 41LR351 \\
\hline 41SM 442 \\
\hline 41CE339 \\
\hline 41CE445 \\
\hline $41 \mathrm{SM} 440$ \\
\hline 41SM442 \\
\hline 41SM444 \\
\hline 41CP239 \\
\hline $41 \mathrm{GG} 31$ \\
\hline 41MR6 \\
\hline $41 \mathrm{CP} 288$ \\
\hline 41TT758 \\
\hline 41FK107 \\
\hline $41 C P 490$ \\
\hline 41CE467 \\
\hline 41CP493 \\
\hline 41CP8, Area A \\
\hline 41CP496 \\
\hline 41HP237 \\
\hline 41HP238 \\
\hline 41HP240 \\
\hline 41WD208 \\
\hline 41SM193 \\
\hline 41SM55 \\
\hline 41HS524 \\
\hline 41PN149 \\
\hline 41RK476 \\
\hline 41NA327 \\
\hline 41SM56 \\
\hline 41SA135 \\
\hline 41CP71 \\
\hline $41 \mathrm{CP} 490$ \\
\hline 41TT891 \\
\hline 41TT892 \\
\hline 41LR351 \\
\hline 41UR10 \\
\hline \\
\hline 41CE19 \\
\hline 41HP78 \\
\hline 41DT16 \\
\hline 41DT52 \\
\hline 41HP102 \\
\hline 41DT1 \\
\hline 41HP105 \\
\hline 41DT80 \\
\hline 41HP105 \\
\hline 41HP78 \\
\hline 41DT80 \\
\hline 41AN19 \\
\hline 41NA144 \\
\hline $41 \mathrm{SY} 81$ \\
\hline 41HS11 \\
\hline 41MR211 \\
\hline 41HS835 \\
\hline 41HE22 \\
\hline 41HE166 \\
\hline 41HE185 \\
\hline 41HE184 \\
\hline 41HE80 \\
\hline 41AN67 \\
\hline 41AN70 \\
\hline 41CE30 \\
\hline 41CE8 6 \\
\hline 41CE19 \\
\hline 41CE19, Late \\
\hline $41 \mathrm{SB} 50$ \\
\hline 41SA89 \\
\hline 41SA94 \\
\hline $415 B 36$ \\
\hline 41NA11 \\
\hline
\end{tabular}


Table 1. East Texas Caddo ceramic sherd database, cont.

\begin{tabular}{|c|c|}
\hline Trinomial & DMUWappliqued-brushed \\
\hline 41CP495 & \\
\hline $41 C P 72$ & \\
\hline 41RA13 & \\
\hline 41CE421 & \\
\hline 41CE423 & \\
\hline 41RA13 & \\
\hline 41CE426 & \\
\hline 41CE429 & \\
\hline 41NA223 & \\
\hline 41CE324 & \\
\hline 41AN87 & 1.50 \\
\hline 41CE309 & 0.10 \\
\hline 41HE337 & \\
\hline 41CE354 & \\
\hline 41RK191 & \\
\hline 41RK197 & \\
\hline 41RK200 & \\
\hline $41 \mathrm{CP} 230$ & \\
\hline 41WD52 & \\
\hline 41FK107 & \\
\hline 41SM195 & \\
\hline $41 \pi T 650$ & \\
\hline 41UR279 & \\
\hline $41 \mathrm{CP} 245$ & \\
\hline 41HS574 & \\
\hline 41BW171 & \\
\hline 41 WD46 & 0.90 \\
\hline 41LR351 & \\
\hline 41SM442 & \\
\hline 41СЕ339 & \\
\hline 41CE445 & \\
\hline $41 \mathrm{SM} 440$ & \\
\hline 41SM442 & \\
\hline 41SM444 & \\
\hline $41 C P 239$ & \\
\hline $41 \mathrm{GG} 31$ & \\
\hline 41MR6 & 1.50 \\
\hline 41 CP288 & \\
\hline $41 T T 758$ & \\
\hline 41FK107 & \\
\hline 41CP490 & \\
\hline 41CE467 & \\
\hline 41CP493 & \\
\hline 41CP8, Area A & \\
\hline $41 C P 496$ & 2.80 \\
\hline 41HP237 & \\
\hline $41 \mathrm{HP} 238$ & \\
\hline $41 \mathrm{HP} 240$ & 0.20 \\
\hline 41WD208 & \\
\hline 41SM193 & \\
\hline 41SM55 & \\
\hline 41HS524 & \\
\hline 41PN149 & \\
\hline 41RK476 & 1.30 \\
\hline 41NA327 & \\
\hline 41SM56 & \\
\hline 41SA135 & \\
\hline 41CP71 & \\
\hline 41CP490 & 2.90 \\
\hline 41тT891 & \\
\hline 41тा892 & \\
\hline 41LR351 & \\
\hline 41UR10 & \\
\hline 41CE19 & \\
\hline 41HP78 & \\
\hline 41DT16 & \\
\hline 41DT52 & \\
\hline 41HP102 & \\
\hline 41DT1 & \\
\hline 41HP105 & \\
\hline 41DT80 & \\
\hline 41HP105 & \\
\hline 41HP78 & \\
\hline $41 \mathrm{DT} 80$ & \\
\hline 41AN19 & \\
\hline 41NA144 & \\
\hline $415 Y 81$ & \\
\hline $41 \mathrm{HS} 11$ & \\
\hline 41MR211 & \\
\hline $41 \mathrm{HS} 835$ & \\
\hline 41HE22 & \\
\hline 41HE166 & \\
\hline 41HE185 & \\
\hline 41HE 184 & \\
\hline 41HE80 & \\
\hline 41AN67 & \\
\hline 41AN70 & \\
\hline 41CE30 & \\
\hline 41CE86 & \\
\hline 41CE19 & \\
\hline 41CE19, Late & \\
\hline 41SB50 & \\
\hline 41SA89 & \\
\hline 41SA94 & \\
\hline $41 \mathrm{SB} 36$ & \\
\hline 41NA11 & \\
\hline
\end{tabular}


4 Journal of Northeast Texas Archaeology 51 (2015)

Table 1. East Texas Caddo ceramic sherd database, cont.

\begin{tabular}{|c|c|}
\hline Trinomial & DMUWneck banded \\
\hline $41 \mathrm{CP} 495$ & 0.30 \\
\hline 41CP72 & \\
\hline 41RA13 & \\
\hline 41CE421 & 0.70 \\
\hline 41CE423 & \\
\hline 41RA13 & \\
\hline 41CE 426 & \\
\hline 41CE429 & 0.60 \\
\hline 41NA223 & 2.10 \\
\hline 41CE324 & \\
\hline 41AN87 & \\
\hline 41CE309 & \\
\hline 41HE337 & \\
\hline 41CE354 & 0.20 \\
\hline 41RK191 & 0.40 \\
\hline 41RK197 & \\
\hline 41RK200 & 0.04 \\
\hline $41 \mathrm{CP} 230$ & 5.70 \\
\hline 41WD52 & 4.30 \\
\hline 41FK107 & \\
\hline 41SM195 & \\
\hline $41 T T 650$ & \\
\hline 41UR279 & \\
\hline $41 C P 245$ & \\
\hline $41 \mathrm{HS} 574$ & \\
\hline 41BW171 & \\
\hline 41WD46 & 2.20 \\
\hline 41LR351 & \\
\hline $41 \mathrm{SM} 442$ & \\
\hline 41СЕ339 & 0.40 \\
\hline 41CE445 & \\
\hline $415 M 440$ & \\
\hline 41SM442 & \\
\hline 41SM444 & \\
\hline $41 \mathrm{CP} 239$ & 1.80 \\
\hline $41 \mathrm{GG} 31$ & 0.30 \\
\hline 41MR6 & \\
\hline $41 C P 288$ & \\
\hline $41 T T 758$ & \\
\hline 41FK107 & \\
\hline $41 \mathrm{CP} 490$ & 5.40 \\
\hline 41CE467 & 1.60 \\
\hline 41CP493 & \\
\hline 41CP8, Area A & \\
\hline $41 \mathrm{CP} 496$ & \\
\hline $41 \mathrm{HP} 237$ & 7.40 \\
\hline $41 \mathrm{HP} 238$ & 21.40 \\
\hline $41 \mathrm{HP} 240$ & 10.00 \\
\hline 41WD208 & 21.80 \\
\hline 41SM193 & \\
\hline 41SM55 & \\
\hline $41 \mathrm{HS} 524$ & \\
\hline 41PN149 & \\
\hline 41RK476 & \\
\hline 41NA327 & \\
\hline 41SM56 & \\
\hline 41SA135 & \\
\hline 41 CP71 & 3.50 \\
\hline $41 C P 490$ & 1.50 \\
\hline 41Tा891 & \\
\hline 41Tा892 & \\
\hline 41LR351 & \\
\hline 41UR10 & \\
\hline 41CE19 & 0.10 \\
\hline $41 \mathrm{HP} 78$ & \\
\hline 41DT16 & \\
\hline 41DT52 & \\
\hline 41 HP102 & \\
\hline 41DT1 & \\
\hline 41HP105 & \\
\hline 41DT80 & \\
\hline 41HP105 & \\
\hline 41HP78 & \\
\hline 41DT80 & \\
\hline 41AN19 & 0.70 \\
\hline 41NA144 & \\
\hline 41SY81 & \\
\hline $41 \mathrm{HS} 11$ & \\
\hline 41MR211 & \\
\hline $41 \mathrm{HS} 835$ & \\
\hline 41HE22 & \\
\hline 41HE166 & \\
\hline 41HE185 & \\
\hline 41HE184 & \\
\hline 41HE8O & \\
\hline 41AN67 & \\
\hline 41AN70 & \\
\hline 41CE30 & \\
\hline 41CE86 & \\
\hline 41CE19 & \\
\hline 41CE19, Late & \\
\hline $41 S B 50$ & \\
\hline 41SA89 & \\
\hline 41SA94 & \\
\hline 41SB36 & \\
\hline 41NA11 & \\
\hline
\end{tabular}


Table 1. East Texas Caddo ceramic sherd database, cont.

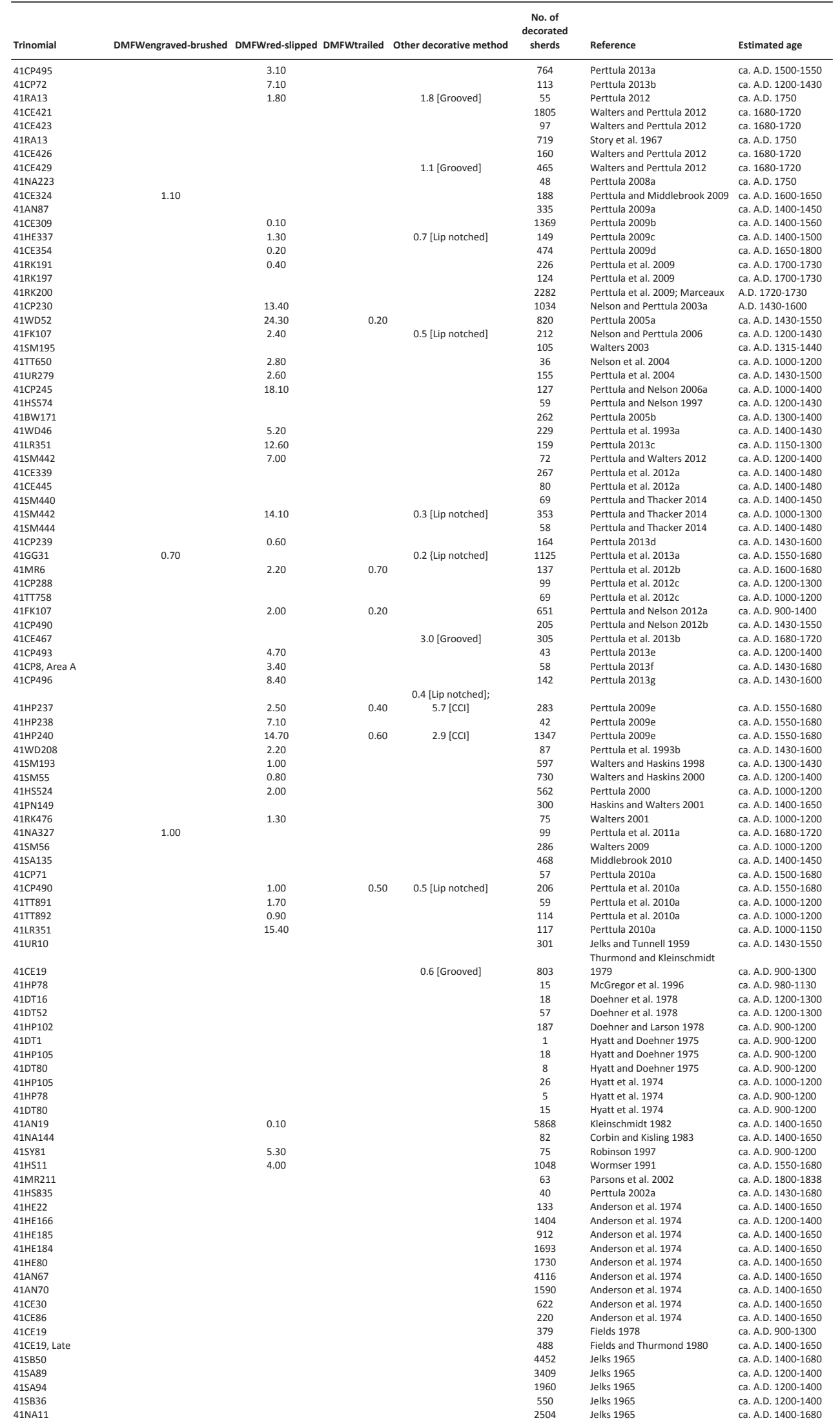


Table 1. East Texas Caddo ceramic sherd database, cont.

\begin{tabular}{|c|}
\hline Trinomial \\
\hline 41SA123 \\
\hline 41SB8 \\
\hline 41SA69 \\
\hline 41SA116 \\
\hline 418W3 \\
\hline 41UR118 \\
\hline 41UR133 \\
\hline 41UR106 \\
\hline 41UR130 \\
\hline 41UR109 \\
\hline 41UR105 \\
\hline 41UR116 \\
\hline 41UR114 \\
\hline 41BW553 \\
\hline $41 T \pi 670$ \\
\hline $41 \mathrm{HS} 240$ \\
\hline 41CP408 \\
\hline 41NA231 \\
\hline 41NA235 \\
\hline 41NA236 \\
\hline 41NA242 \\
\hline 41NA285 \\
\hline 41NA338 \\
\hline 41NA21 \\
\hline 41NA304 \\
\hline 41NA303 \\
\hline 41HP106 \\
\hline 41RK170 \\
\hline 41CP304 \\
\hline 41HS15 \\
\hline 41NA49 \\
\hline 41HE70 \\
\hline 41VN6 \\
\hline 41DT1 \\
\hline 41SM73 \\
\hline 41SM74 \\
\hline 41SM76 \\
\hline 41SM82 \\
\hline 41SM87 \\
\hline $415 \mathrm{M} 89$ \\
\hline $41 \mathrm{SM} 90$ \\
\hline 41SM91 \\
\hline 41HE22 \\
\hline 41CE39 \\
\hline 41WD529 \\
\hline 41WD51 \\
\hline 41HE114 \\
\hline 41HP175 \\
\hline 41RA5 \\
\hline 41BW5 \\
\hline 41HS16 \\
\hline 41SA83 \\
\hline 41LR1 \\
\hline 41RA8 \\
\hline $41 \mathrm{GG} 33$ \\
\hline 41RK3 \\
\hline 41BW169 \\
\hline 41BW716 \\
\hline 41PN175 \\
\hline 41СР20 \\
\hline 41RK19 \\
\hline 41RK30 \\
\hline 41RK39 \\
\hline 41SM54 \\
\hline $41 \mathrm{HP} 1$ \\
\hline $41 \Pi 13$ \\
\hline 41DT11 \\
\hline 41DT21 \\
\hline 41DT54 \\
\hline 41DT63 \\
\hline 41WD73 \\
\hline 41WD482 \\
\hline 41WD495 \\
\hline 41WD538 \\
\hline 41WD450 \\
\hline 41WD503 \\
\hline 41WD109 \\
\hline 41UR142 \\
\hline 41UR136 \\
\hline 41HE139 \\
\hline 41AN38 \\
\hline 41SM243 \\
\hline 41RK4 \\
\hline 41SM 325 \\
\hline 41TT769 \\
\hline Hickory Creek \#2 \\
\hline 41WD75 \\
\hline 41WD524 \\
\hline 41RA48 \\
\hline 41WD74 \\
\hline 41RR15 \\
\hline 41RR204 \\
\hline 41 CP55 \\
\hline 41UR271 \\
\hline 41CE19, Vil \\
\hline 41CE19, Md \\
\hline$y_{1}$ \\
\hline
\end{tabular}


Table 1. East Texas Caddo ceramic sherd database, cont.

\begin{tabular}{|c|}
\hline Trinomial \\
\hline 41SA123 \\
\hline 41SB8 \\
\hline 41SA69 \\
\hline 41SA116 \\
\hline 41BW3 \\
\hline 41UR118 \\
\hline 41UR133 \\
\hline 41UR106 \\
\hline 41UR130 \\
\hline 41UR109 \\
\hline 41UR105 \\
\hline 41UR116 \\
\hline 41UR114 \\
\hline 41BW553 \\
\hline $41 T T 670$ \\
\hline $41 \mathrm{HS} 240$ \\
\hline 41CP408 \\
\hline 41NA231 \\
\hline 41NA235 \\
\hline 41NA236 \\
\hline 41NA242 \\
\hline 41NA285 \\
\hline 41NA338 \\
\hline 41NA21 \\
\hline 41NA304 \\
\hline 41NA303 \\
\hline 41HP106 \\
\hline 41RK170 \\
\hline $41 C P 304$ \\
\hline 41HS15 \\
\hline 41NA49 \\
\hline 41HE70 \\
\hline 41VN6 \\
\hline 41DT1 \\
\hline 41SM73 \\
\hline 41SM74 \\
\hline 41SM76 \\
\hline $41 \mathrm{SM} 82$ \\
\hline 41SM87 \\
\hline 41SM89 \\
\hline $41 \mathrm{SM} 90$ \\
\hline 41SM91 \\
\hline 41HE22 \\
\hline 41CE39 \\
\hline 41WD529 \\
\hline 41WD51 \\
\hline 41HE114 \\
\hline 41HP175 \\
\hline 41RA5 \\
\hline 41BW5 \\
\hline $41 \mathrm{HS} 16$ \\
\hline 41SA83 \\
\hline 41LR1 \\
\hline 41RA8 \\
\hline $41 \mathrm{GG} 33$ \\
\hline 41RK3 \\
\hline 41BW169 \\
\hline 41BW716 \\
\hline 41PN175 \\
\hline $41 \mathrm{CP} 20$ \\
\hline 41RK19 \\
\hline 41RK30 \\
\hline 41RK39 \\
\hline 41SM54 \\
\hline $41 \mathrm{HP} 1$ \\
\hline $41 \pi T 13$ \\
\hline 41DT11 \\
\hline 41DT21 \\
\hline 41DT54 \\
\hline 41DT63 \\
\hline 41WD73 \\
\hline 41WD482 \\
\hline 41WD495 \\
\hline 41WD538 \\
\hline 41WD450 \\
\hline 41WD503 \\
\hline 41WD109 \\
\hline 41UR142 \\
\hline 41UR136 \\
\hline 41HE139 \\
\hline 41AN38 \\
\hline 41SM243 \\
\hline 41RK4 \\
\hline 41SM325 \\
\hline $41 T T 769$ \\
\hline Hickory Creek \#2 \\
\hline 41WD75 \\
\hline 41WD524 \\
\hline 41RA48 \\
\hline 41WD74 \\
\hline 41RR15 \\
\hline 41RR204 \\
\hline 41CP55 \\
\hline 41UR271 \\
\hline 41CE19, Village \\
\hline 41CE19, Md. A \\
\hline 41CE19, Md. B \\
\hline
\end{tabular}


8 Journal of Northeast Texas Archaeology 51 (2015)

Table 1. East Texas Caddo ceramic sherd database, cont.

\begin{tabular}{|c|}
\hline Trinomial \\
\hline 41SA123 \\
\hline 41SB8 \\
\hline 41SA69 \\
\hline 41SA116 \\
\hline 41BW3 \\
\hline 41UR118 \\
\hline 41UR133 \\
\hline 41UR106 \\
\hline 41UR130 \\
\hline 41UR109 \\
\hline 41UR105 \\
\hline 41UR116 \\
\hline 41UR114 \\
\hline 41BW553 \\
\hline $41 \pi 670$ \\
\hline $41 \mathrm{HS} 240$ \\
\hline 41CP408 \\
\hline 41NA231 \\
\hline 41NA235 \\
\hline 41NA236 \\
\hline 41NA242 \\
\hline 41NA285 \\
\hline 41NA338 \\
\hline 41NA21 \\
\hline 41NA304 \\
\hline 41NA303 \\
\hline 41HP106 \\
\hline 41RK170 \\
\hline 41СР304 \\
\hline 41HS15 \\
\hline 41NA49 \\
\hline 41HE70 \\
\hline $41 \mathrm{VN} 6$ \\
\hline 41DT1 \\
\hline 41SM73 \\
\hline 41SM74 \\
\hline 41SM76 \\
\hline 41SM82 \\
\hline 41SM87 \\
\hline 415M89 \\
\hline 41SM90 \\
\hline 41SM91 \\
\hline 41HE22 \\
\hline 41CE39 \\
\hline 41WD529 \\
\hline 41WD51 \\
\hline 41HE114 \\
\hline 41HP175 \\
\hline 41RA5 \\
\hline 41BW5 \\
\hline 41HS16 \\
\hline 41SA83 \\
\hline 41LR1 \\
\hline 41RA8 \\
\hline $41 \mathrm{GG} 33$ \\
\hline 41RK3 \\
\hline 41BW169 \\
\hline 41BW716 \\
\hline 41PN175 \\
\hline 41CP20 \\
\hline 41RK19 \\
\hline 41RK30 \\
\hline 41RК 39 \\
\hline 41SM54 \\
\hline $41 \mathrm{HP} 1$ \\
\hline $41 \pi 13$ \\
\hline 41DT11 \\
\hline 41DT21 \\
\hline 41DT54 \\
\hline 41DT63 \\
\hline 41WD73 \\
\hline 41WD482 \\
\hline 41WD495 \\
\hline 41WD538 \\
\hline 41WD450 \\
\hline 41WD503 \\
\hline 41WD109 \\
\hline 41UR142 \\
\hline 41UR136 \\
\hline 41HE139 \\
\hline 41AN38 \\
\hline 41SM243 \\
\hline 41RK4 \\
\hline 41SM325 \\
\hline $41 \pi 7769$ \\
\hline Hickory Creek \#2 \\
\hline 41WD75 \\
\hline 41WD524 \\
\hline 41RA48 \\
\hline 41WD74 \\
\hline 41RR15 \\
\hline 41RR204 \\
\hline 41CP55 \\
\hline 41UR271 \\
\hline 41CE19, Village \\
\hline 41CE19, Md. A \\
\hline 41CE19, Md. B \\
\hline
\end{tabular}


Table 1. East Texas Caddo ceramic sherd database, cont.

\begin{tabular}{|c|}
\hline Trinomial \\
\hline 41SA123 \\
\hline 41SB8 \\
\hline 41SA69 \\
\hline 41SA116 \\
\hline 41BW3 \\
\hline 41UR118 \\
\hline 41UR133 \\
\hline 41UR106 \\
\hline 41UR130 \\
\hline 41UR109 \\
\hline 41UR105 \\
\hline 41UR116 \\
\hline 41UR114 \\
\hline 41BW553 \\
\hline $41 T \pi 670$ \\
\hline $41 \mathrm{HS} 240$ \\
\hline 41CP408 \\
\hline 41NA231 \\
\hline 41NA235 \\
\hline 41NA236 \\
\hline 41NA242 \\
\hline 41NA285 \\
\hline 41NA338 \\
\hline 41NA21 \\
\hline 41NA304 \\
\hline 41NA303 \\
\hline 41HP106 \\
\hline 41RK170 \\
\hline 41CP304 \\
\hline $41 \mathrm{HS} 15$ \\
\hline 41NA49 \\
\hline 41HE70 \\
\hline 41VN6 \\
\hline 41DT1 \\
\hline 41SM73 \\
\hline $415 M 74$ \\
\hline 41SM76 \\
\hline $415 M 82$ \\
\hline 41SM87 \\
\hline 41SM89 \\
\hline 41SM90 \\
\hline 41SM91 \\
\hline 41HE22 \\
\hline $41 \mathrm{CE} 39$ \\
\hline 41WD529 \\
\hline 41WD51 \\
\hline 41HE114 \\
\hline 41HP175 \\
\hline 41RA5 \\
\hline $41 \mathrm{BW} 5$ \\
\hline $41 \mathrm{HS} 16$ \\
\hline 41SA83 \\
\hline 41LR1 \\
\hline 41RA8 \\
\hline $41 \mathrm{GG} 33$ \\
\hline 41RK3 \\
\hline 41BW169 \\
\hline 41BW716 \\
\hline 41PN175 \\
\hline 41CP20 \\
\hline 41RK19 \\
\hline 41RK30 \\
\hline 41RK39 \\
\hline 41SM54 \\
\hline 41HP1 \\
\hline $41 \pi T 13$ \\
\hline 41DT11 \\
\hline 41DT21 \\
\hline 41DT54 \\
\hline 41DT63 \\
\hline 41 WD73 \\
\hline 41WD482 \\
\hline 41WD495 \\
\hline 41WD538 \\
\hline 41WD450 \\
\hline 41WD503 \\
\hline 41WD109 \\
\hline 41UR142 \\
\hline 41UR136 \\
\hline 41HE139 \\
\hline 41AN38 \\
\hline $415 \mathrm{SM} 24$ \\
\hline 41RK4 \\
\hline 41SM 325 \\
\hline $41 T T 769$ \\
\hline Hickory Creek \#2 \\
\hline 41WD75 \\
\hline 41WD524 \\
\hline 41RA48 \\
\hline 41WD74 \\
\hline 41RR15 \\
\hline 41RR204 \\
\hline 41CP55 \\
\hline 41UR271 \\
\hline 41CE19, Village \\
\hline 41CE19, Md. A \\
\hline 41CE19, Md. B \\
\hline
\end{tabular}


10 Journal of Northeast Texas Archaeology 51 (2015)

Table 1. East Texas Caddo ceramic sherd database, cont.

\begin{tabular}{|c|c|c|c|c|c|}
\hline Trinomial & Tgrog & Tbone & Tshell & Tgrog-bone & Psandy \\
\hline 41AN1 & & & & & \\
\hline 41AN2 & & & & & \\
\hline 41AN8 & & & & & \\
\hline 41AN23 & & & & & \\
\hline 41AN32 & & & & & \\
\hline 41CE3 & & & & & \\
\hline 41CE4 & & & & & \\
\hline 41CE8 & & & & & \\
\hline 41AN38 & 82.70 & 4.30 & & 12.70 & 0.60 \\
\hline 41LR170 & 92.20 & 2.00 & & 5.90 & \\
\hline 41LR186 & 76.60 & 6.40 & & 12.80 & 4.30 \\
\hline 41LR187 & 74.10 & 8.10 & 0.40 & 17.10 & 0.40 \\
\hline 41NA27 & 82.70 & & & 17.30 & \\
\hline $41 \mathrm{CP} 10$ & & & & & \\
\hline 41DT80 & 78.80 & 0.90 & 5.90 & & 14.40 \\
\hline 41DT124 & 89.60 & 8.60 & 0.40 & & 1.40 \\
\hline 41RR48 & 72.00 & 0.90 & & 20.60 & 6.50 \\
\hline 41LR60 & 75.00 & 4.00 & 16.00 & 2.00 & 8.00 \\
\hline 41LR39 & 74.40 & 1.10 & 0.30 & 23.70 & 0.60 \\
\hline 41RK19 & & & & & \\
\hline 41RK21 & & & & & \\
\hline 41RK32 & & & & & \\
\hline 41RK36 & & & & & \\
\hline 41RK39 & & & & & \\
\hline 41RK214 & 80.80 & 2.00 & & 16.80 & \\
\hline $41 \mathrm{GG} 33$ & 75.60 & 10.50 & & 14.00 & \\
\hline $41 \mathrm{GG} 33$ & 45.40 & 27.30 & & 27.30 & \\
\hline $415 Y 92$ & & & & & \\
\hline 41AN51 & 86.30 & 13.70 & & & \\
\hline $41 \mathrm{CP} 71$ & & & & & \\
\hline $41 \mathrm{HS} 74$ & 75.80 & 20.00 & & & 4.20 \\
\hline 41BW5 & 26.70 & 2.20 & 68.60 & 0.20 & 2.30 \\
\hline 41LR2 & 51.40 & 6.20 & 10.20 & & 32.20 \\
\hline 41RR14 & 42.40 & 0.30 & 40.00 & 8.10 & 9.40 \\
\hline 41RR16 & 63.20 & 3.00 & 4.60 & 29.10 & \\
\hline 41RR16 & & & 100.00 & & \\
\hline 41RR11 & & & & & \\
\hline 41RR11 & & & 100.00 & & \\
\hline 41RR236 & 39.70 & & 60.30 & & \\
\hline $41 R R 248$ & & & 100.00 & & \\
\hline $41 R R 290$ & 61.90 & & 38.10 & & \\
\hline $41 \mathrm{BW} 3$ & 88.70 & 1.30 & 1.80 & 8.20 & \\
\hline $41 T T 672$ & 83.30 & 3.30 & & 6.70 & 6.70 \\
\hline 41NA49 & 45.80 & 5.90 & 0.10 & 48.20 & 0.30 \\
\hline $41 \mathrm{HO} 5 \mathrm{O}$ & 77.40 & & & 22.60 & \\
\hline $41 T T 653$ & 64.10 & & & 35.90 & \\
\hline 41RR16 & 92.90 & 7.10 & & & \\
\hline 41RR16 & & & 100.00 & & \\
\hline 41CE19 & & & & & \\
\hline 41DT16 & 72.40 & 8.60 & 0.50 & 5.90 & \\
\hline 41MR2 & & & & & \\
\hline 41UR11 & & & & & \\
\hline 41MR12 & & & & & \\
\hline 41MR1 & & & & & \\
\hline 41UR30 & 74.70 & 4.40 & & 20.90 & \\
\hline 41TT653 & 77.90 & 8.20 & & 4.10 & 2.10 \\
\hline 41RK214 & 85.50 & 0.90 & & 0.90 & 12.80 \\
\hline 41RK215 & 100.00 & & & & 10 \\
\hline 41RK216 & 95.50 & & & & 4.50 \\
\hline 41NA235 & & & & & \\
\hline 41NA236 & & & & & \\
\hline 41NA244 & & & & & \\
\hline 41NA248 & & & & & \\
\hline 41NA264 & & & & & \\
\hline 41NA285 & & & & & \\
\hline 41NA243 & & & & & \\
\hline 41NA247 & & & & & \\
\hline 41CP257 & & & & & \\
\hline 41CP272 & & & & & \\
\hline 41FK107 & & & & & \\
\hline 41TT804 & & & & & \\
\hline $41 T T 310$ & 72.00 & 28.00 & & & \\
\hline $41 \mathrm{HS} 573$ & 48.00 & 29.00 & 0.30 & 21.00 & 0.30 \\
\hline $41 \mathrm{HS} 574$ & 57.00 & 20.00 & & 17.00 & \\
\hline $41 \mathrm{HS} 843$ & 38.00 & 36.00 & 6.60 & 16.00 & \\
\hline $41 \mathrm{HS} 844$ & 63.00 & 11.00 & 0.60 & 25.00 & \\
\hline $41 \mathrm{HS} 846$ & 70.00 & 13.00 & & 16.00 & 0.60 \\
\hline $41 \mathrm{HS} 588$ & 58.90 & 3.40 & 2.60 & 35.00 & \\
\hline 41FT425 & 64.00 & 19.00 & 0.10 & 13.00 & 3.00 \\
\hline 41DT11 & 63.00 & 13.90 & 3.00 & 13.80 & 4.60 \\
\hline 41NA60 & 89.40 & 3.40 & 0.40 & 5.30 & 1.50 \\
\hline 41TT372 & 83.20 & 1.10 & & 14.60 & 1.10 \\
\hline 41CE354 & 100.00 & & & & \\
\hline 41CE354 & 96.00 & 0.90 & & 2.70 & 1.30 \\
\hline 41LR11 & 69.20 & & & 27.20 & 3.50 \\
\hline 41LR31 & & & & & \\
\hline 41MX5 & 29.00 & 14.00 & & & 49.00 \\
\hline 41HO91 & 84.60 & 5.10 & & 7.70 & 2.60 \\
\hline 41CE461 & & & & & \\
\hline 41NA15 & 80.30 & 8.20 & 0.40 & 10.70 & 0.40 \\
\hline $41 \mathrm{HO} 263$ & 72.90 & 6.30 & & 18.80 & 2.10 \\
\hline 41NA321 & 80.60 & 6.50 & & 12.90 & \\
\hline
\end{tabular}


Table 1. East Texas Caddo ceramic sherd database, cont.

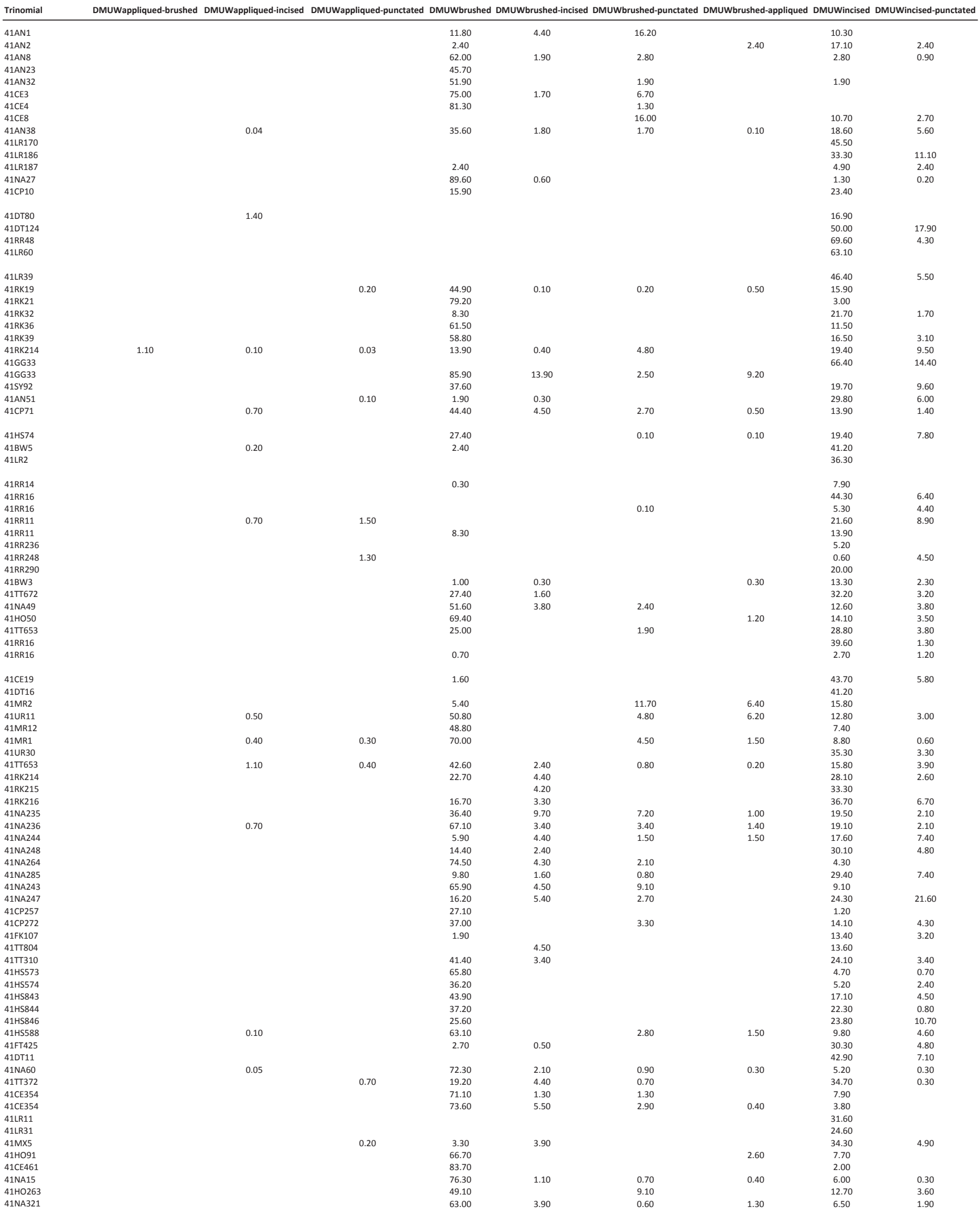


Table 1. East Texas Caddo ceramic sherd database, cont.

\begin{tabular}{|c|c|c|c|c|c|c|c|c|c|}
\hline Trinomial & DMUWneck banded & DMUWpinched & DMUWtool punctated & DMUWfingernail punctated & DMUWcircular punctated & DMUWcane punctated & DMUWridged $\mathrm{D}$ & DMFeng.-punct. & DMFWengraved \\
\hline 41AN1 & 8.80 & 2.90 & 7.30 & & & & & & 38.30 \\
\hline 41AN2 & 17.10 & & 7.30 & 7.30 & & & & & 39.00 \\
\hline 41AN8 & 0.90 & 0.90 & & 1.90 & & & & & 25.90 \\
\hline $41 \mathrm{AN} 23$ & & 2.90 & & & & & & & 51.40 \\
\hline 41AN32 & & & 1.90 & & & & & & 42.30 \\
\hline $41 \mathrm{CE} 3$ & & 6.70 & & & & & & & 10.00 \\
\hline 41CE4 & 2.70 & & & & & & & & 18.70 \\
\hline $41 C E 8$ & 18.70 & 4.00 & 2.70 & & & & & & 45.30 \\
\hline $41 \mathrm{AN} 38$ & 0.10 & 0.40 & 10.40 & 0.50 & & 0.20 & & 0.40 & 20.70 \\
\hline 41LR170 & & & & 9.10 & & 18.20 & & & 27.30 \\
\hline 41LR186 & & 11.10 & & 11.10 & & & & & 33.30 \\
\hline $\begin{array}{l}411 \mathrm{R} 187 \\
41 \mathrm{NA27}\end{array}$ & 0.03 & 0.01 & $\begin{array}{l}9.80 \\
0.60\end{array}$ & $\begin{array}{l}22.00 \\
0.80\end{array}$ & & & & & 29.30 \\
\hline $41 \mathrm{CP} 10$ & 0.03 & 0.01 & & & & & & & $\begin{array}{l}8.00 \\
35.20\end{array}$ \\
\hline 41DT80 & 8.50 & & & 47.90 & & & & 2.80 & 12.70 \\
\hline 41DT124 & & & 5.40 & 8.90 & 5.40 & & & 3.60 & 7.10 \\
\hline 41RR48 & & & 4.30 & & 4.30 & & & & 4.30 \\
\hline 41LR60 & & & 7.80 & 2.00 & & & & & 2.00 \\
\hline 41LR39 & & 2.60 & 13.20 & 0.90 & & & & 0.40 & 14.00 \\
\hline 41RK19 & 0.30 & 0.10 & 26.70 & 3.50 & & & & & 7.20 \\
\hline 41RK21 & & 0.70 & 8.90 & 0.70 & & & & & 6.70 \\
\hline & 1.70 & & 23.30 & 3.30 & & & & & 3.30 \\
\hline 41RK36 & & & 19.20 & & & & & & 7.70 \\
\hline $41 \mathrm{RK} 39$ & & & 10.30 & & & & & & 11.30 \\
\hline 41RK214 & & 0.60 & 7.50 & 22.50 & 0.30 & 1.80 & & & 11.30 \\
\hline $41 \mathrm{GG} 33$ & & 2.90 & 7.20 & 2.00 & & & & & 6.40 \\
\hline $41 \mathrm{GG} 33$ & & & 1.30 & & & & & & 2.50 \\
\hline $415 Y 92$ & & & & & & & & & 19.50 \\
\hline 41AN51 & & 1.90 & 20.80 & 8.60 & 2.30 & 0.10 & & & 16.30 \\
\hline $41 \mathrm{CP} 71$ & 0.50 & & 3.40 & & & & & 0.70 & 18.70 \\
\hline $41 \mathrm{HS74}$ & & & & 4.30 & & & & & 22.80 \\
\hline 418W5 & & & 2.20 & 0.20 & & & & & 52.80 \\
\hline 41LR2 & 16.50 & & & 10.00 & & & & & 24.40 \\
\hline 41RR14 & 0.80 & & & & & & & & 51.60 \\
\hline 41RR16 & & & & & & & & & 13.20 \\
\hline 41RR16 & 35.30 & & & & & & & & 21.70 \\
\hline 41RR11 & & & & & & & & & 31.30 \\
\hline 41RR11 & 8.30 & & & & & & & & 25.00 \\
\hline $41 R R 236$ & & & & 13.80 & & & & & 10.30 \\
\hline $\begin{array}{l}41 R R 248 \\
417 B 290\end{array}$ & 79.60 & & & & & & & & 5.70 \\
\hline $\begin{array}{l}411 \mathrm{R} 2290 \\
41 \mathrm{BW} 3\end{array}$ & & & 2.00 & 0.30 & 100 & & & 100 & 20.00 \\
\hline $41 \Pi 7672$ & & & $\begin{array}{l}2.00 \\
16.10\end{array}$ & $\begin{array}{l}0.30 \\
4.80\end{array}$ & 1.00 & & & 1.00 & $\begin{array}{l}43.00 \\
14.50\end{array}$ \\
\hline 41NA49 & & 0.60 & & 4.80 & & & & 0.10 & $\begin{array}{l}14.50 \\
13.40\end{array}$ \\
\hline $41 \mathrm{HO} 0$ & & & & 1.20 & & & & 0.10 & 7.10 \\
\hline $41 \Pi 653$ & & & & & & & 1.90 & & 17.30 \\
\hline 41RR16 & & & & & & & & & 0.60 \\
\hline 41RR16 & 23.60 & & & & & & & & 29.80 \\
\hline 41CE19 & 0.40 & 0.40 & 0.40 & 3.70 & & & 0.30 & & 10.00 \\
\hline 41DT16 & & & & 11.80 & & & 5.90 & & 29.40 \\
\hline 41MR2 & 0.60 & & 5.40 & & & 1.60 & & 0.10 & 37.90 \\
\hline 41UR11 & & 2.40 & 1.00 & 0.80 & & & & 0.20 & 9.10 \\
\hline 41MR12 & & & & & & & & & 32.80 \\
\hline 41MR1 & 0.10 & & & & & & 1.50 & & 7.40 \\
\hline 41UR30 & & 2.00 & 9.20 & 8.50 & 2.00 & & & & 34.70 \\
\hline $41 \Pi 653$ & 0.90 & & 9.40 & 1.00 & 0.20 & 0.10 & & & 4.70 \\
\hline 41RK214 & & & 15.70 & & & & & & 9.60 \\
\hline 41RK215 & & & 16.70 & 16.70 & & & & & 29.20 \\
\hline 41RK216 & & & 13.30 & & 10.00 & & & 6.70 & 6.70 \\
\hline 41NA235 & 0.50 & & 8.70 & 1.00 & & 1.00 & & & 10.80 \\
\hline 41NA236 & & 0.70 & 1.40 & 0.70 & & & & & \\
\hline 41NA244 & & & 7.40 & 13.20 & & 3.00 & & & 23.50 \\
\hline 41NA248 & & & 4.80 & 4.80 & & & & & \\
\hline $\begin{array}{l}\text { 41NA264 } \\
\end{array}$ & & & 2.10 & 4.30 & 2.10 & & & & 6.40 \\
\hline $\begin{array}{l}\text { 411NA28245 } \\
\text { 4NA24 }\end{array}$ & & 1.60 & 8.20 & 7.40 & & 2.30 & & & $\begin{array}{l}32.80 \\
680\end{array}$ \\
\hline 41NA247 & & & 8.10 & 5.40 & & 2.30 & & & $\begin{array}{l}6.80 \\
16.20\end{array}$ \\
\hline 41CP257 & 1.20 & & 13.00 & 13.00 & & & & & $\begin{array}{l}20.20 \\
23.50\end{array}$ \\
\hline $41 \mathrm{CP} 272$ & 2.20 & & 6.50 & 4.40 & & 1.10 & & & 15.20 \\
\hline 41FK107 & & & 7.00 & 54.10 & & 3.80 & & & 11.40 \\
\hline $41 \Pi 804$ & & & 4.50 & 45.40 & & & & & 18.20 \\
\hline & & & 3.40 & 17.20 & & & & & 6.90 \\
\hline 41HS573 & & & 2.90 & 1.80 & & & 1.10 & & 12.30 \\
\hline $41 \mathrm{HS} 574$ & & 3.30 & 23.90 & 1.40 & & & & & 21.00 \\
\hline $41 \mathrm{HS} 843$ & & & & & & & & & 29.30 \\
\hline 41HS844 & & 0.80 & 5.00 & 10.70 & & & & & 20.70 \\
\hline $41 \mathrm{H} 5846$ & 0.60 & 0.60 & 8.30 & 6.00 & & & & & 19.10 \\
\hline 41HS588 & & 0.80 & 5.80 & 1.40 & & & 0.40 & & 9.70 \\
\hline 41FT425 & & 1.60 & 8.10 & 27.00 & & & & & 29.70 \\
\hline 41DT11 & & 0.60 & 1.30 & 4.60 & & & & & 35.70 \\
\hline 41NA60 & 0.10 & & 1.50 & 0.10 & 0.10 & & & & 12.70 \\
\hline $41 \Pi \pi 372$ & & & 8.10 & 8.80 & & & & & 23.20 \\
\hline 41CE354 & 1.30 & & & & & & & & 14.50 \\
\hline 41CE354 & 0.20 & & 0.60 & & & & & & 11.60 \\
\hline 41LR11 & & & 5.30 & 26.30 & & & & & 26.30 \\
\hline 41LR31 & $15 \mathrm{co}$ & & & 8.80 & & & & & 14.00 \\
\hline $\begin{array}{l}\text { 41MX55 } \\
411091\end{array}$ & 15.60 & 260 & 1.20 & 3.10 & & & & & $\begin{array}{l}19.10 \\
15.40\end{array}$ \\
\hline $\begin{array}{l}\text { 41HO91 } \\
\text { 41CE461 }\end{array}$ & & 2.60 & & & 2.00 & & & & $\begin{array}{l}15.40 \\
10.20\end{array}$ \\
\hline 41NA15 & & 0.20 & 1.90 & & & & & 0.10 & 11.40 \\
\hline $41 \mathrm{HO} 263$ & 3.60 & & 9.10 & 3.60 & 1.90 & & & & $\begin{array}{l}7.30 \\
130\end{array}$ \\
\hline 41NA321 & & & 5.80 & & 0.60 & & & & 13.00 \\
\hline
\end{tabular}


Table 1. East Texas Caddo ceramic sherd database, cont.

\begin{tabular}{|c|c|c|c|}
\hline Trinomial & DMFWengraved-brushed & DMFWred-slipped & DMFWtrailed \\
\hline 41AN1 & & & \\
\hline 41AN2 & 4.90 & & \\
\hline 41AN8 & & & \\
\hline $41 \mathrm{AN} 23$ & & & \\
\hline 41AN32 & & & \\
\hline 41CE3 & & & \\
\hline 41CE4 & & & \\
\hline 41CE8 & & & \\
\hline 41AN38 & 0.10 & 0.70 & \\
\hline 41LR170 & & & \\
\hline 41LR186 & & & \\
\hline 41LR187 & & 26.80 & \\
\hline 41NA27 & Trace & & \\
\hline $\begin{array}{l}41 \mathrm{CP} 10 \\
\end{array}$ & Tace & & \\
\hline 41DT80 & & 4.20 & \\
\hline 41DT124 & & 1.80 & \\
\hline 41RR48 & & 13.00 & \\
\hline 41LR60 & & 13.70 & \\
\hline & & & \\
\hline 41LR39 & & 14.90 & \\
\hline 41RK19 & & 0.50 & \\
\hline 41RK21 & & 0.70 & \\
\hline 41RK32 & & 1.70 & \\
\hline 41RK36 & & & \\
\hline 41RK39 & & & \\
\hline 41RK214 & & 0.80 & 0.03 \\
\hline $41 \mathrm{GG} 33$ & & & \\
\hline $41 \mathrm{GG} 33$ & & & \\
\hline $415 Y 92$ & & & \\
\hline 41AN51 & & 11.30 & \\
\hline 41CP71 & & 6.20 & 0.50 \\
\hline $41 \mathrm{HS} 74$ & & & \\
\hline 41BW5 & & 0.50 & \\
\hline 41LR2 & & 10.00 & \\
\hline & & & \\
\hline 41RR14 & & 25.40 & \\
\hline 41RR16 & & 17.40 & \\
\hline 41RR16 & & 6.10 & 1.40 \\
\hline 41RR11 & & 18.70 & \\
\hline 41RR11 & & 30.60 & \\
\hline 41RR236 & & 58.60 & \\
\hline 41RR248 & & 4.50 & \\
\hline 41RR290 & & 30.00 & 10.00 \\
\hline $41 \mathrm{BW} 3$ & & 2.30 & 30.80 \\
\hline $41 T 672$ & & & \\
\hline 41NA49 & & & 1.40 \\
\hline $41 \mathrm{HO} 50$ & & & \\
\hline $41 \pi 653$ & & 3.80 & \\
\hline 41RR16 & & 44.20 & \\
\hline 41RR16 & & 42.80 & 0.60 \\
\hline 41CE19 & & 0.60 & \\
\hline 41DT16 & & 5.90 & \\
\hline 41MR2 & & 7.80 & \\
\hline 41UR11 & & 3.50 & 1.40 \\
\hline 41MR12 & & 7.80 & 0.60 \\
\hline 41MR1 & & & \\
\hline 41UR30 & & & \\
\hline $41 \Pi 653$ & & 4.60 & \\
\hline 41RK214 & & & \\
\hline 41RK215 & & & \\
\hline 41RK216 & & & \\
\hline 41NA235 & & 1.00 & \\
\hline 41NA236 & & & \\
\hline 41NA244 & & & \\
\hline 41NA248 & & & \\
\hline 41NA264 & & & \\
\hline 41NA285 & & & \\
\hline 41NA243 & 2.30 & & \\
\hline 41NA247 & & & \\
\hline $41 C P 257$ & & 16.00 & \\
\hline $41 C P 272$ & & 10.90 & \\
\hline 41FK107 & & 0.60 & \\
\hline 41TT804 & & 4.50 & \\
\hline $41 \Pi 310$ & & & \\
\hline 41HS573 & & & \\
\hline 41HS574 & & & \\
\hline $41 \mathrm{HS} 843$ & & 2.40 & \\
\hline $41 \mathrm{HS} 844$ & & 0.80 & \\
\hline $41 \mathrm{HS} 846$ & & 2.40 & \\
\hline $41 \mathrm{HS} 588$ & & & 0.10 \\
\hline 41FT425 & & & \\
\hline 41DT11 & & 7.80 & \\
\hline 41NA60 & 0.90 & & 0.10 \\
\hline $41 T \pi 372$ & & & \\
\hline 41CE354 & & & \\
\hline 41CE354 & & 0.20 & \\
\hline $\begin{array}{l}\text { 41LR11 } \\
\end{array}$ & & 10.50 & \\
\hline 41LR31 & & 50.90 & \\
\hline 41MX5 & & 2.10 & 2.50 \\
\hline 41HO91 & & & \\
\hline 41CE 461 & & & 2.00 \\
\hline 41NA15 & 0.10 & & \\
\hline $41 \mathrm{HO} 263$ & & & \\
\hline 41NA321 & & 0.60 & \\
\hline
\end{tabular}


14 Journal of Northeast Texas Archaeology 51 (2015)

Table 1. East Texas Caddo ceramic sherd database, cont.

\begin{tabular}{|c|c|}
\hline Trinomial & Tgrog \\
\hline 41NA336 & 92.40 \\
\hline $41 \mathrm{CS} 14$ & 89.90 \\
\hline 41SM273, Block I & 61.80 \\
\hline 41SM273, BI. III & 70.20 \\
\hline 41SM273, BI. II & 68.70 \\
\hline $41 T T 396$ & 81.90 \\
\hline 41TT400 & 72.40 \\
\hline 41DT6 & 53.40 \\
\hline 41DT16 & 58.40 \\
\hline $41 T T 769$ & 26.30 \\
\hline $41 \mathrm{TT} 13$ & \\
\hline $41 \mathrm{BW} 600$ & 98.50 \\
\hline $41 \mathrm{HO} 211$ & 77.40 \\
\hline $41 \mathrm{HO} 214$ & 74.00 \\
\hline 41SM272 & 97.10 \\
\hline $41 \mathrm{CP} 314$ & \\
\hline $41 \mathrm{CP} 317$ & \\
\hline 41CР304 & 95.00 \\
\hline $41 C P 315$ & 89.00 \\
\hline 41SM272 & 76.00 \\
\hline 41SM273 & \\
\hline $415 Y 100$ & 52.20 \\
\hline 16SA101 & 40.10 \\
\hline 16SA17 & 39.00 \\
\hline $165 \mathrm{~S} 204$ & 76.20 \\
\hline $165 \mathrm{~A} 62$ & \\
\hline 16SA30B & \\
\hline 16SA37B & \\
\hline 16SA37A & \\
\hline 16SA30A & \\
\hline 41TT110 & \\
\hline 41UR1 & \\
\hline 41UR3 & \\
\hline 41UR13 & \\
\hline 41UR14 & \\
\hline 41UR18 & \\
\hline 41WD16 & \\
\hline $41 \mathrm{CP} 8$ & \\
\hline 41 CP14 & \\
\hline 41FK4 & \\
\hline $41 \mathrm{MX} 6$ & \\
\hline $41 \mathrm{MX8}$ & \\
\hline $41 T T 4$ & \\
\hline $41 \mathrm{TT} 6$ & \\
\hline $41 \Pi T 17$ & \\
\hline $41 T T 28$ & \\
\hline $41 \pi T 52$ & \\
\hline $41 \mathrm{CP} 15$ & \\
\hline $41 \mathrm{HS} 1$ & \\
\hline $41 \mathrm{HS} 10$ & \\
\hline $41 \mathrm{HS} 11$ & \\
\hline 41MR6 & \\
\hline 41MR13 & \\
\hline 41MR31 & \\
\hline $41 \mathrm{M} \times 22$ & \\
\hline $41 \mathrm{TT} 18$ & \\
\hline 41TT151 & \\
\hline 41UR15 & \\
\hline $41 \mathrm{CP} 3$ & \\
\hline $41 \mathrm{CP} 71$ & \\
\hline $41 \mathrm{CP} 55$ & 97.80 \\
\hline 41LR2 & 85.50 \\
\hline $41 \mathrm{GG5}$ & 91.80 \\
\hline $41 \mathrm{GG} 50$ & 89.40 \\
\hline 41BW3, VP 1 & 85.70 \\
\hline $41 \mathrm{BW} 4$ & 97.20 \\
\hline 41RK19 & 88.70 \\
\hline & \\
\hline $41 \mathrm{BW} 2$ & 84.90 \\
\hline $41 \pi T 12$ & 76.40 \\
\hline $41 T T 11$ & 72.70 \\
\hline 41NA317 & 78.00 \\
\hline CE299 & 79.90 \\
\hline 41SM404 & 92.00 \\
\hline 41RK240 & 72.10 \\
\hline 41RK242 & 76.80 \\
\hline 41RK243 & 74.60 \\
\hline 41UR106 & \\
\hline 41UR106B & \\
\hline UR109 & \\
\hline 41UR118 & \\
\hline 41UR129 & \\
\hline 41UR133, Saddle & \\
\hline 41LR297 & 62.50 \\
\hline $415 Y 323$ & 11.90 \\
\hline 41RK557 & 42.90 \\
\hline $41 \mathrm{HS} 269$ & \\
\hline PN175 & 31.70 \\
\hline HS12 & 58.10 \\
\hline $41 \mathrm{CP} 408$ & 40.60 \\
\hline 41AN21 & 94.30 \\
\hline 41 CE39 & 94.60 \\
\hline NA321 & \\
\hline 41CE20 & 85.70 \\
\hline 41CE48 & 69.90 \\
\hline 41CE29: & 93.60 \\
\hline
\end{tabular}


Table 1. East Texas Caddo ceramic sherd database, cont.

\begin{tabular}{|c|c|c|}
\hline Trinomial & DMUWappliqued-brushed & DMUWappliqued-incised \\
\hline 41NA336 & & \\
\hline $41 \mathrm{cs} 14$ & & \\
\hline 41SM273, Block I & & \\
\hline 41SM273, BI. III & & \\
\hline 41SM273, BI. II & & \\
\hline 41тT396 & & \\
\hline 41TT400 & & \\
\hline 41DT6 & & \\
\hline 41DT16 & & \\
\hline 41TT769 & & \\
\hline 41TT13 & & \\
\hline 41BW600 & & \\
\hline 41HO211 & & \\
\hline $41 \mathrm{HO} 214$ & 1.70 & \\
\hline 41SM272 & & \\
\hline 41CP314 & & \\
\hline 41СР317 & & 1.50 \\
\hline 41CP304 & & 列 \\
\hline 41CP315 & & \\
\hline 41SM272 & & \\
\hline 41SM273 & & \\
\hline $41 \mathrm{SY} 100$ & & \\
\hline 16SA101 & & \\
\hline 16SA17 & & \\
\hline 16SA204 & & \\
\hline 16SA62 & 0.04 & 0.10 \\
\hline 16SА30B & & \\
\hline 16SA37B & & \\
\hline 16SA37A & & \\
\hline 16SA30A & & \\
\hline 41TT110 & & \\
\hline 41UR1 & & \\
\hline 41UR3 & & \\
\hline 41UR13 & & \\
\hline 41UR14 & & \\
\hline 41UR18 & & 0.10 \\
\hline 41WD16 & & \\
\hline $41 \mathrm{CP} 8$ & & 0.10 \\
\hline 41CP14 & & \\
\hline 41FK4 & & \\
\hline 41MX6 & & \\
\hline $41 \mathrm{M} \times 8$ & & \\
\hline 41TT4 & & \\
\hline 41тT6 & & \\
\hline 41тT17 & & 0.80 \\
\hline $41 T T 28$ & & \\
\hline 41TT52 & & \\
\hline 41CP15 & & \\
\hline $41 \mathrm{HS} 1$ & & \\
\hline $41 \mathrm{HS} 10$ & & \\
\hline 41HS11 & & \\
\hline 41MR6 & & \\
\hline 41MR13 & & \\
\hline 41MR31 & & \\
\hline 41MX22 & & \\
\hline 41TT18 & & \\
\hline 41TT151 & & \\
\hline 41UR15 & & \\
\hline 41СР3 & & \\
\hline 41CP71 & & 0.50 \\
\hline 41CP55 & & \\
\hline 41LR2 & & \\
\hline $41 \mathrm{GG5}$ & & \\
\hline 41GG50 & & \\
\hline 41BW3, VP 1 & 0.60 & \\
\hline 41BW4 & 0.70 & 0.40 \\
\hline 41RK19 & & 0.05 \\
\hline 41BW2 & & 1.20 \\
\hline 41TT12 & & 0.30 \\
\hline 41тT11 & & 0.60 \\
\hline 41NA317 & & \\
\hline 41CE299 & & \\
\hline 41SM404 & & \\
\hline 41RK240 & & \\
\hline 41RK242 & & \\
\hline 41RK243 & & \\
\hline 41UR106 & & \\
\hline 41UR106B & & 0.50 \\
\hline 41UR109 & & 0.30 \\
\hline 41UR118 & & \\
\hline 41UR129 & & \\
\hline 41UR133, Saddle & & \\
\hline 41LR297 & & \\
\hline $415 Y 323$ & & \\
\hline 41RK557 & & \\
\hline 41HS269 & & \\
\hline 41PN175 & & 0.40 \\
\hline $41 \mathrm{HS} 12$ & & \\
\hline 41CP408 & & 0.30 \\
\hline 41AN21 & & 0.70 \\
\hline 41CE39 & & \\
\hline & & \\
\hline 41CE20 & & \\
\hline 41CE48 & & \\
\hline 41CE293 & & \\
\hline
\end{tabular}


16 Journal of Northeast Texas Archaeology 51 (2015)

Table 1. East Texas Caddo ceramic sherd database, cont.

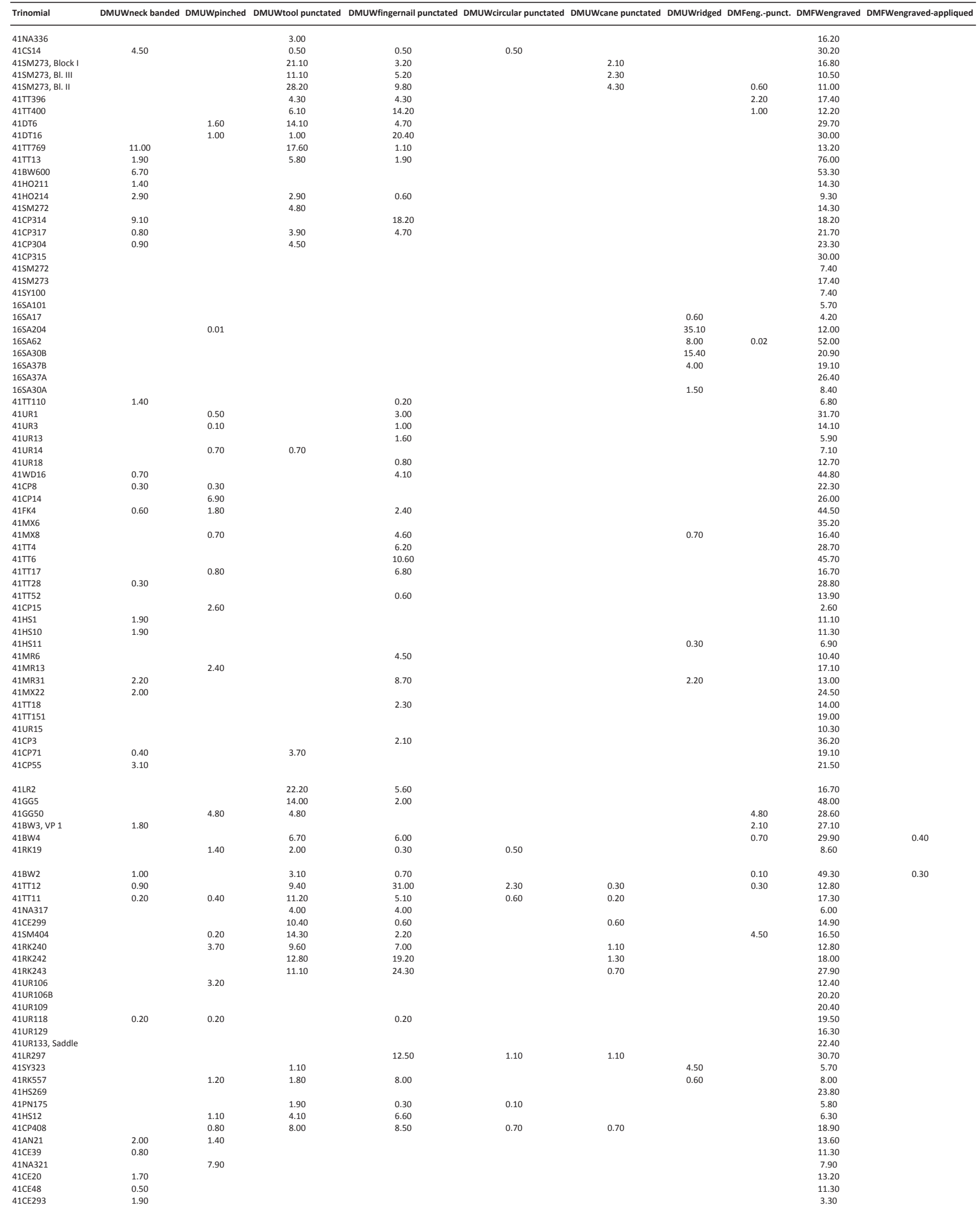


Table 1. East Texas Caddo ceramic sherd database, cont.

\begin{tabular}{|c|c|c|}
\hline Trinomial & DMFWengraved-brushed & DMFWred-slipped \\
\hline 41NA336 & & \\
\hline $41 \mathrm{CS} 14$ & & 2.00 \\
\hline 41SM273, Block I & & 4.20 \\
\hline 41SM273, Bl. III & & 0.60 \\
\hline 41SM273, Bl. II & & 1.20 \\
\hline $41 T \pi 396$ & & 6.50 \\
\hline $41 T \pi 400$ & & 8.20 \\
\hline 41DT6 & & 14.10 \\
\hline 41DT16 & & 13.60 \\
\hline $41 T \pi 769$ & & 18.70 \\
\hline $41 \Pi 13$ & & 1.90 \\
\hline 41BW600 & & \\
\hline $41 \mathrm{HO} 211$ & & \\
\hline $41 \mathrm{HO} 214$ & & \\
\hline 41SM272 & & \\
\hline $41 \mathrm{CP} 314$ & & 12.10 \\
\hline $41 \mathrm{CP} 317$ & & 6.20 \\
\hline 41CP304 & & 8.00 \\
\hline $41 C P 315$ & & 6.70 \\
\hline 41SM272 & & 3.70 \\
\hline 41SM273 & & 3.50 \\
\hline $415 Y 100$ & & \\
\hline 16SA101 & & \\
\hline 16SA17 & & \\
\hline $165 \mathrm{~A} 204$ & & \\
\hline $16 \mathrm{SA} A 2$ & & \\
\hline $16 \mathrm{SA} 30 \mathrm{~B}$ & & \\
\hline 16SA37B & & \\
\hline 16SA37A & & \\
\hline 16SA30A & & \\
\hline $41 T \pi 110$ & & 29.50 \\
\hline 41UR1 & & 9.00 \\
\hline 41UR3 & & 0.70 \\
\hline 41UR13 & & \\
\hline 41UR14 & & 1.70 \\
\hline 41UR18 & & 3.00 \\
\hline 41WD16 & & 4.10 \\
\hline $41 \mathrm{CP} 8$ & & 2.20 \\
\hline $41 \mathrm{CP} 14$ & & \\
\hline 41FK4 & & 4.30 \\
\hline $41 \mathrm{M} \times 6$ & & 3.30 \\
\hline $41 \mathrm{M} \times 8$ & & 1.30 \\
\hline 41TT4 & & 3.10 \\
\hline $41 T T 6$ & & 4.30 \\
\hline $41 \Pi \pi 17$ & & 6.10 \\
\hline $41 T T 28$ & & \\
\hline $41 \pi 552$ & & 7.50 \\
\hline $41 \mathrm{CP} 15$ & & 28.20 \\
\hline 41HS1 & & 1.90 \\
\hline $41 \mathrm{HS} 10$ & & \\
\hline $41 \mathrm{HS} 11$ & & \\
\hline 41MR6 & & 1.50 \\
\hline 41MR13 & & \\
\hline 41MR31 & & 2.20 \\
\hline $41 \mathrm{M} \times 22$ & & 2.00 \\
\hline $41 T T 18$ & & 9.30 \\
\hline $41 \pi T 151$ & & \\
\hline 41UR15 & & \\
\hline $41 C P 3$ & & 4.30 \\
\hline $41 \mathrm{CP} 71$ & & 4.70 \\
\hline 41CP55 & & \\
\hline 41LR2 & & 22.20 \\
\hline $41 \mathrm{GG5}$ & & \\
\hline $41 \mathrm{GG} 50$ & & \\
\hline 41BW3, VP 1 & & 4.80 \\
\hline $41 \mathrm{BW} 4$ & & 3.40 \\
\hline 41RK19 & & 0.10 \\
\hline $41 \mathrm{BW} 2$ & & 2.00 \\
\hline $41 \pi 12$ & & 3.10 \\
\hline $41 \pi T 11$ & & 11.20 \\
\hline 41NA317 & & \\
\hline 41CE299 & & \\
\hline 41SM404 & & 6.50 \\
\hline 41RK240 & & \\
\hline 41RK242 & & 1.30 \\
\hline 41RK243 & & \\
\hline 41UR106 & & 6.30 \\
\hline 41UR106B & & 1.60 \\
\hline 41UR109 & & 0.30 \\
\hline 41UR118 & & \\
\hline 41UR129 & & 2.00 \\
\hline 41UR133, Saddle & & \\
\hline 41LR297 & & \\
\hline $41 S Y 323$ & & 2.30 \\
\hline 41RK557 & & \\
\hline $41 \mathrm{HS} 269$ & & \\
\hline 41PN175 & & 0.20 \\
\hline $41 \mathrm{HS} 12$ & & 1.40 \\
\hline 41CP408 & 0.10 & 5.40 \\
\hline 41AN21 & & 1.40 \\
\hline 41СЕЗ9 & & \\
\hline 41NA321 & & \\
\hline $41 \mathrm{CE} 20$ & & \\
\hline 41CE48 & & \\
\hline 41CE293 & & \\
\hline
\end{tabular}


Table 1. East Texas Caddo ceramic sherd database, cont.

\begin{tabular}{|c|c|c|c|c|c|c|c|c|c|c|c|c|c|c|c|c|c|c|}
\hline Trinomial & Tgrog & Tbone & Tshell & Tgrog-bone & Psandy & Freduced & Foxidized & d Finc-oxidized & Fred-oxidized & Rfdirect & Rfinverted & Rfeverted & Lfrounded & Lfflat & Lfrounded-folded Lfbeveled & Pred & Pwhite & DMUWappliqued \\
\hline $41 \mathrm{AG} 22$ & 92.00 & 1.60 & & 6.40 & & 9.40 & 9.40 & 18.80 & 61.90 & 66.70 & & 33.30 & 92.90 & 7.10 & & & 1.20 & 0.60 \\
\hline 41CE62 & 71.20 & 4.50 & & 24.20 & & 5.70 & 26.20 & 13.90 & 54.20 & & & & & & & & & \\
\hline 41NA6 & 85.10 & 6.60 & & 7.70 & 0.50 & 12.60 & 13.70 & 17.10 & 56.60 & 71.40 & 14.30 & 14.30 & 66.70 & 22.20 & 11.10 & & & 0.30 \\
\hline 41NA15 & 79.20 & 7.00 & & 13.90 & & 16.70 & 7.60 & 19.70 & 56.10 & 69.20 & & 30.80 & 64.30 & 21.40 & 14.30 & & 0.50 & 0.50 \\
\hline 41NA44 & 64.20 & 21.70 & & 10.70 & 3.30 & 21.40 & 20.30 & 10.90 & 47.30 & 62.50 & 1.60 & 35.90 & 71.60 & 13.60 & 14.80 & & & 0.70 \\
\hline 41NA54 & 73.50 & 10.80 & & 15.70 & & 13.10 & 4.80 & 15.50 & 66.70 & & & & & & & & & \\
\hline 41NA21 & 27.60 & 42.80 & 0.40 & 28.80 & 0.50 & 22.60 & 12.20 & 13.90 & 51.20 & 52.20 & 8.70 & 39.10 & 80.00 & 14.50 & 5.70 & & 0.50 & 0.10 \\
\hline 41NA22 & 57.20 & 20.60 & & 20.60 & 1.60 & 10.30 & 16.60 & 9.20 & 63.90 & 78.10 & & 21.90 & 83.30 & 7.30 & 9.10 & & 0.10 & 0.10 \\
\hline 41NA23 & 84.50 & 5.50 & & 9.50 & 0.30 & 11.50 & 22.90 & 16.70 & 48.90 & 73.50 & & 26.50 & 64.40 & 17.80 & 17.80 & & & 0.30 \\
\hline 41NA111 & 59.60 & 22.20 & & 18.20 & & 9.10 & 11.10 & 17.20 & 62.60 & & & & & & & & & 0.50 \\
\hline 41NA183 & 64.30 & 13.20 & 1.00 & 21.40 & & 24.00 & 12.50 & 5.20 & 58.30 & & & & & & & & & 0.40 \\
\hline 41NA206 & 56.40 & 31.50 & 0.80 & 10.20 & 1.00 & 34.10 & 22.00 & 8.30 & 35.80 & 77.50 & & 22.50 & 67.50 & 6.10 & 26.50 & & 0.02 & 0.20 \\
\hline 41NA67 & 75.50 & 6.30 & & 18.10 & & 29.70 & 6.30 & 10.90 & 53.10 & 62.50 & & 37.50 & 85.70 & 2.40 & 11.90 & & & \\
\hline 41SA94 & 58.50 & 18.10 & 0.70 & 22.90 & & 13.60 & 7.80 & 9.60 & 68.90 & 88.90 & 3.00 & 8.10 & 53.30 & 20.00 & 26.70 & & & 0.20 \\
\hline 41SA25 & 1.30 & 75.20 & 12.90 & 5.50 & 5.40 & & & & & 68.80 & 3.10 & 28.10 & 73.00 & 8.10 & 18.90 & & & \\
\hline 41BW3, Mound & & & & & & & & & & & & & & & & & & 9.90 \\
\hline $415 A 25$ & 1.70 & 79.20 & 10.80 & 8.60 & 0.10 & & & & & 18.90 & 11.70 & 69.40 & & & & & & \\
\hline 41WD577 & 76.20 & 1.00 & & 18.60 & 4.10 & & & & & 89.50 & & 10.50 & 38.50 & 42.30 & 19.20 & & & 2.00 \\
\hline $415 Y 43$ & 51.50 & 48.40 & & & & & & & & & & & & & & & & 0.20 \\
\hline $415 Y 279$ & 24.00 & 76.00 & & & & & & & & & & & & & & & & 1.10 \\
\hline $415 Y 280$ & 18.30 & 81.70 & & & & & & & & & & & & & & & & 1.50 \\
\hline 41LR2 & 72.40 & 11.70 & 15.90 & & & & & & & & & & & & & & & 5.40 \\
\hline $41 \mathrm{SY} 41$ & 69.80 & 30.20 & & & & & & & & & & & & & & & & 1.60 \\
\hline $41 \mathrm{SY} 45$ & 53.70 & 46.30 & & & & & & & & & & & & & & & & \\
\hline $415 Y 27$ & 17.00 & 83.00 & & & & & & & & & & & & & & & & 4.50 \\
\hline 41LR2, NMNH & 68.00 & 13.40 & 18.60 & & & & & & & & & & & & & & & 5.20 \\
\hline $41 \mathrm{GG} 69$ & 84.40 & 15.60 & & & & & & & & & & & & & & & & 0.40 \\
\hline $41 \mathrm{FN} 1$ & 49.30 & 10.30 & 40.40 & & & & & & & & & & & & & & & \\
\hline 41LR1 & 71.40 & 10.00 & 18.50 & & & & & & & & & & & & & & & 3.10 \\
\hline 41WD3 & 99.40 & 0.60 & & & & & & & & & & & & & & & & 11.70 \\
\hline 41TT851 & 64.00 & 10.30 & & 25.50 & & & & & & & & & & & & & & 2.40 \\
\hline 41TT852 & 45.30 & 8.30 & & 46.40 & & & & & & & & & & & & & & 3.00 \\
\hline 41TT853 & 65.30 & 1.90 & & 32.80 & & & & & & & & & & & & & & 0.60 \\
\hline 41WD6 & 95.20 & 4.80 & & & & & & & & & & & & & & & & 15.80 \\
\hline 41WD1 & 96.90 & 3.10 & & & & & & & & & & & & & & & & 4.80 \\
\hline
\end{tabular}

Table 1. East Texas Caddo ceramic sherd database, cont.

\begin{tabular}{|c|c|c|c|c|c|c|c|c|c|}
\hline Trinomial & DMUWappliqued-brushed & DMUWappliqued-incised & DMUWappliqued-punctated & DMUWbrushed & DMUWbrushed-incised $\mathrm{D}$ & DMUWbrushed-punctated & DMUWbrushed-appliqued & DMUWincised & DMUWincised-punctated \\
\hline $41 \mathrm{AG} 22$ & & & & 87.70 & 0.60 & 1.80 & 0.60 & 1.20 & 1.20 \\
\hline 41CE62 & & & & 90.50 & & & & 5.40 & \\
\hline 41NAG & & & 0.10 & 78.80 & 1.60 & 2.10 & 0.70 & 4.30 & 0.30 \\
\hline 41NA15 & & & & 60.50 & 2.20 & 3.20 & 1.60 & 2.70 & 0.50 \\
\hline 41NA44 & 1.40 & & 0.60 & 55.70 & 5.80 & 5.10 & & 7.20 & 1.90 \\
\hline 41NA54 & 1.10 & & & 72.00 & 8.50 & 3.20 & & 5.80 & 0.50 \\
\hline 41NA21 & & & 0.03 & 68.90 & 0.30 & 0.40 & & 6.90 & 0.20 \\
\hline 41NA22 & 3.80 & 0.03 & 0.70 & 77.40 & 1.60 & 3.60 & & 3.40 & 0.10 \\
\hline 41NA23 & 0.80 & & 0.70 & 76.90 & 1.00 & 1.90 & & 6.30 & 0.70 \\
\hline 41NA111 & 1.90 & & 0.90 & 79.70 & 1.90 & 2.30 & & 1.40 & \\
\hline 41NA183 & & & 1.30 & 58.00 & 1.70 & 1.30 & & 10.90 & 3.40 \\
\hline 41NA206 & 0.50 & & 0.20 & 52.60 & 2.80 & 0.60 & & 12.30 & 1.40 \\
\hline 41NA67 & & & & 16.20 & & 1.00 & & 24.80 & 10.00 \\
\hline 41SA94 & 0.60 & & & 46.00 & 1.00 & 2.60 & & 12.60 & 5.00 \\
\hline 41SA25 & & 0.10 & & 0.60 & 0.10 & & & 35.60 & 0.80 \\
\hline 41BW3, Mound & & & & 12.40 & & & & 36.60 & \\
\hline 41SA25 & & & & & & & & 29.10 & 3.40 \\
\hline 41WD577 & & & 5.00 & 1.00 & 1.00 & 2.00 & & 21.80 & 6.00 \\
\hline $41 \mathrm{SY} 43$ & & 0.10 & & 66.30 & 3.00 & 1.80 & 1.00 & 9.00 & 0.60 \\
\hline $415 Y 279$ & & 1.10 & & 52.80 & & & 2.20 & 20.90 & 1.10 \\
\hline $415 Y 280$ & & & & 55.70 & 6.30 & 0.40 & & 17.30 & 0.30 \\
\hline 41LR2 & & & & 1.20 & & & & 15.00 & \\
\hline $415 Y 41$ & & & & 45.20 & 3.20 & 6.50 & & 9.00 & 9.50 \\
\hline 41SҮ45 & & & & 62.70 & 5.90 & 4.90 & & 8.60 & 3.20 \\
\hline $415 Y 27$ & & & & 87.20 & & 0.80 & 0.80 & 1.70 & \\
\hline 41LR2, NMNH & & & 0.40 & 0.40 & & & & 10.90 & 1.50 \\
\hline $41 \mathrm{GG} 69$ & & 0.20 & & 13.30 & 3.50 & 1.80 & & 24.40 & 21.90 \\
\hline 41FN1 & & & & 3.60 & & & & 28.60 & \\
\hline 41LR1 & & 0.30 & 0.60 & 3.90 & 0.60 & 0.30 & & 31.90 & 1.40 \\
\hline 41WD3 & & & & 21.10 & 8.80 & & 4.10 & 2.90 & \\
\hline 41TT851 & & & 0.70 & 11.50 & & 0.50 & 0.20 & 18.10 & 2.70 \\
\hline 41TT852 & & 0.30 & 0.70 & 32.60 & & 1.50 & 0.70 & 17.70 & 2.50 \\
\hline 41TT853 & & 0.10 & 0.40 & 43.20 & & 2.70 & 2.50 & 15.00 & 4.60 \\
\hline 41WD6 & & & 1.40 & 3.40 & 0.50 & & & 12.50 & 1.00 \\
\hline 41WD1 & & & 1.00 & 5.80 & & & & 43.30 & 1.00 \\
\hline
\end{tabular}


Table 1. East Texas Caddo ceramic sherd database, cont.

\begin{tabular}{|c|c|c|c|c|c|c|c|c|c|c|}
\hline Trinomial & DMUWneck banded & DMUWpinched & DMUWtool punctated & DMUWfingernail punctated & DMUWcircular punctated & DMUWcane punctated & DMUWridged & DMFeng.-punct. & DMFWengraved & DMFWengraved-appliqued \\
\hline $41 \mathrm{AG} 22$ & & & & & & & & & 4.30 & \\
\hline 41CE62 & & & & & & & & & 3.40 & \\
\hline 41NAG & & 1.50 & & & & & & & 7.70 & \\
\hline 41NA15 & 0.50 & 0.50 & & & & & & & 23.20 & \\
\hline 41NA44 & 0.10 & 1.10 & & & & & & 0.10 & 12.00 & \\
\hline 41NA54 & & 1.10 & & & & & & & 5.80 & \\
\hline 41NA21 & 0.20 & & & & & & & & 10.50 & \\
\hline 41NA22 & & 0.30 & & & & & & & 6.40 & \\
\hline 41NA23 & 0.70 & 0.20 & & & & & & & 8.00 & \\
\hline 41NA111 & 0.50 & 0.50 & & & & & & & 6.90 & \\
\hline 41NA183 & & & & & & & & 0.40 & 8.40 & \\
\hline 41NA206 & 0.10 & 0.40 & & & & & & & 22.30 & \\
\hline 41NA67 & & & 21.50 & 2.40 & & & & & 23.80 & \\
\hline 41SA94 & & 0.30 & & & & & & & 24.90 & \\
\hline 41SA25 & & & & & & & & & 59.30 & \\
\hline 41BW3, Mound & 2.00 & & & & & & & & 8.10 & \\
\hline 41SA25 & & & 2.00 & 0.50 & 0.20 & & & & 64.80 & \\
\hline 41WD577 & & & 6.90 & 8.90 & 6.90 & & & & 20.00 & \\
\hline $41 \mathrm{~S} Y 43$ & & & 3.40 & 0.10 & & & 0.30 & 0.30 & 13.50 & \\
\hline $415 Y 279$ & & & 4.40 & & & & 4.40 & & 9.90 & \\
\hline $415 Y 280$ & & & 2.80 & 0.10 & & & 6.80 & 0.10 & 5.00 & \\
\hline 41LR2 & 3.00 & & 3.00 & 4.20 & 1.20 & 0.60 & & & 51.50 & \\
\hline $41 \mathrm{~S} 441$ & & & 5.30 & 2.10 & 1.10 & & & & 16.00 & \\
\hline 41SY45 & & & 4.90 & 0.50 & & & & & 9.30 & \\
\hline $41 \mathrm{SY} 27$ & & & & & & & 0.80 & & 4.10 & \\
\hline 41LR2, NMNH & 7.90 & 0.40 & 2.20 & 9.00 & 1.10 & 0.40 & & & 36.90 & \\
\hline $41 \mathrm{GG} 69$ & & 0.50 & 12.30 & 1.60 & 3.00 & 0.20 & 0.20 & & 16.10 & 0.20 \\
\hline 41FN1 & & & 3.60 & 1.80 & & & & & 8.90 & \\
\hline 41LR1 & 0.90 & 0.30 & 6.80 & 5.90 & & & & & 33.30 & \\
\hline 41WD3 & 12.30 & & 0.60 & 2.90 & & & & & 30.40 & \\
\hline 41ТT851 & 0.50 & 5.60 & 16.40 & 17.90 & & & & & 23.30 & \\
\hline 41TT852 & 0.30 & 1.20 & 11.80 & 5.90 & & & & & 21.90 & \\
\hline 41ТT853 & 0.50 & 2.70 & 6.20 & 4.60 & & & & & 17.10 & \\
\hline 41WD6 & 30.30 & & 3.80 & 0.50 & & & & & 25.00 & \\
\hline 41WD1 & & 1.00 & 5.80 & 3.90 & 1.00 & & & & 14.40 & \\
\hline
\end{tabular}

Table 1. East Texas Caddo ceramic sherd database, cont.

\begin{tabular}{|c|c|c|c|c|c|c|c|}
\hline Trinomial & DMFWengraved-brushed & DMFWred-slipped & d DMFWtrailed & Other decorative method & $\begin{array}{c}\text { No. of } \\
\text { decorated } \\
\text { sherds }\end{array}$ & Reference & Estimated age \\
\hline 41AG22 & & & & & 163 & Marceaux 2011 & ca. A.D. $1680-1730$ \\
\hline 41CE62 & & & & & 148 & Marceaux 2011 & Ca. A.D. $1680-1730$ \\
\hline 41NA6 & 0.70 & & & 0.1 [Grooved] & 673 & Marceaux 2011 & Ca. A.D. $1680-1730$ \\
\hline 41NA15 & 1.60 & & & 1.6 [Grooved] & 185 & Marceaux 2011 & Ca. A.D. $1680-1730$ \\
\hline 41NA44 & 0.30 & & 0.10 & 0.8 [Grooved] & 1812 & Marceaux 2011 & Ca. A.D. $1680-1730$ \\
\hline 41NA54 & & & & & 189 & Marceaux 2011 & Ca. A.D. $1680-1730$ \\
\hline 41NA21 & 0.10 & & 0.02 & $\begin{array}{cc}0.03 \text { [Lip notched] } \\
0.4 \text { [grooved]; } 0.2 \text { [lip }\end{array}$ & 9819 & Marceaux 2011 & ca. A.D. $1680-1730$ \\
\hline 41NA22 & 0.10 & & & 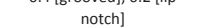 & 2874 & Marceaux 2011 & Ca. A.D. $1680-1730$ \\
\hline 41NA23 & 0.10 & & & 0.6 [grooved] & 2301 & Marceaux 2011 & Ca. A.D. $1680-1730$ \\
\hline 41NA111 & & & & 0.5 [grooved] & 217 & Marceaux 2011 & Ca. A.D. $1680-1730$ \\
\hline 41NA183 & 0.40 & & & & 238 & Marceaux 2011 & Ca. A.D. $1680-1730$ \\
\hline & & & & 0.4 [grooved]; 0.1 [lip & & & \\
\hline 41NA206 & 0.02 & & 0.02 & notch] & 4156 & Marceaux 2011 & Ca. A.D. $1680-1730$ \\
\hline 41NA67 & & & 0.50 & & 210 & Marceaux 2011 & Ca. A.D. $1680-1730$ \\
\hline 41SA94 & & & & 0.1 [lip notched] & 1195 & Marceaux 2011 & ca. A.D. $1500-1700$ \\
\hline 41SA25 & & & & & 1940 & Marceaux 2011 & ca. A.D. $1720-1770$ \\
\hline 41BW3, Mound & & 8.10 & 7.30 & 6.3 [roughened] & 6198 & Perttula 2014b & ca. A.D. $1400-1690$ \\
\hline 41SA25 & & & & & 441 & Corbin et al. 1990 & ca. A.D. $1720-1770$ \\
\hline 41WD577 & & 18.90 & & & 101 & Perttula and Gilmore 1988 & ca. A.D. $1200-1430$ \\
\hline $41 S Y 43$ & & & 0.30 & & 873 & Selden and Perttula 2014 & ca. A.D. $1400-1600$ \\
\hline $415 Y 279$ & & & 1.10 & $\begin{array}{c}1.1 \text { [Lip notched] } \\
0.1 \text { \{lmpressed\}; } 0.1\end{array}$ & 91 & Selden and Perttula 2014 & ca. A.D. $1500-1600$ \\
\hline $415 Y 280$ & & & 3.10 & [Stamped] & 753 & Selden and Perttula 2014 & ca. A.D. $1500-1600$ \\
\hline 41LR2 & & 14.40 & 0.60 & & 167 & Perttula et al. 2015 & ca. A.D. $1100-1700$ \\
\hline 41SY41 & & & & 0.5 [grooved] & 188 & Perttula 2014h & ca. A.D. $1400-1500$ \\
\hline $41 \mathrm{SY} 45$ & & & & & 185 & Perttula 2014i & ca. A.D. $1400-1500$ \\
\hline $415 Y 27$ & & & & & 258 & Perttula and Selden 2014 & $\begin{array}{l}\text { ca. A.D. } 1450-1550 \\
\text { ca. A.D. } 1100-1300 \text {, }\end{array}$ \\
\hline 41LR2, NMNH & & 17.60 & 4.10 & 1.1 [lip notched]; $0.7, \mathrm{cCl}$ & 266 & Perttula et al. 2015 & $1600-1740$ \\
\hline $41 \mathrm{GG} 69$ & & $\begin{array}{l}17.50 \\
0.50\end{array}$ & & & $\begin{array}{l}200 \\
570\end{array}$ & Perttula 2015a & ca. A.D. $1300-1400$ \\
\hline $41 \mathrm{FN} 1$ & & 51.80 & 1.80 & & 56 & Perttula 2015b & $\begin{array}{l}\text { 1680-1730 } \\
\text { ca. A.D. } 1100-1300,\end{array}$ \\
\hline 41LR1 & & 10.20 & 0.30 & 0.3 [lip notched] & 354 & Perttula 2015c & $1680-1740$ \\
\hline 41WD3 & & 5.30 & & & 171 & Perttula 2015d & ca. A.D. $1430-1680$ \\
\hline 41TT851 & & & & & 408 & Fields et al. 2014 & ca. A.D. $1250-1325$ \\
\hline $41 T 7852$ & & & & & 745 & Fields et al. 2014 & ca. A.D. $1425-1500$ \\
\hline $41 T T 853$ & & & & $\begin{array}{c}0.1 \text { [lip notched] } \\
1.0 \text { [lip notched]; } 1.4\end{array}$ & 787 & Fields et al. 2014 & ca. A.D. $1400-1500$ \\
\hline 41 WD 6 & & 3.40 & & [grooved] & 208 & Perttula 2015e & ca. A.D. $1430-1600$ \\
\hline 41WD1 & & 17.30 & & 1.0 [lip notched] & 104 & Perttula $2015 \mathrm{f}$ & ca. A.D. $1200-1400$ \\
\hline
\end{tabular}




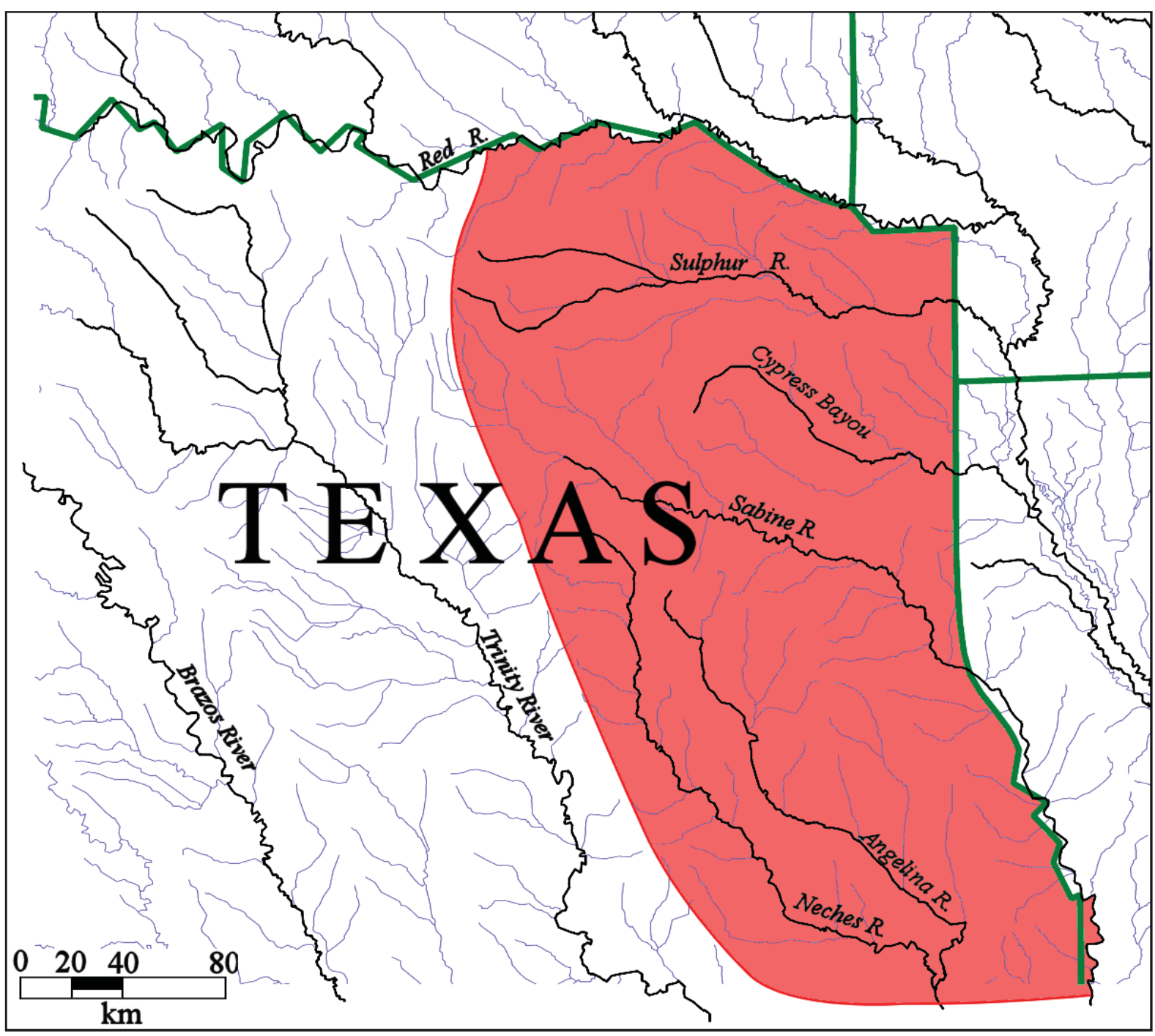

Figure 1. East Texas Caddo area.

circular punctated, cane punctated, and ridged) and fine wares (engraved, engraved-punctated, engravedappliqued, engraved-brushed, red-slipped, and trailed), as well as other decorative methods (grooved, lip notched, corn cob impressed [CCI], cord impressed, fabric impressed, roughened, trailed-incised, painted, stamped); (g) number of decorated sherds; (h) reference; and (i) estimated age of the site and/or component assemblages, as best as can be determined from published analyses and reported calibrated radiocarbon dates. The focus on methods of decoration in the assemblages is in recognition of the fact that their differences across sites and assemblages provide an indication of regional variation in ceramic assemblages, and the broad categories of decoration "are less subject to inter-observer variation in classification than finer type designations" (Peeples and Roberts 2013:3003).

\section{INITIAL INTERPRETATIONS}

In this section, I review several interpretive findings from the ceramic sherd database regarding East Texas Caddo ceramics. These findings have barely plumbed the depths of the ceramic sherd database, but constitute a beginning effort at the identification of similarities in Caddo ceramic assemblages that likely have a basis in regional patterns of interaction within social networks (e.g., Mills et al. 2013) between differ- 
ent Caddo communities. That is to say, the residents of different settlements of Caddo peoples with similar ceramic assemblages (however measured) were most likely to have interacted more frequently with each other than they did with other Caddo settlements with quite different and dissimilar ceramic assemblages.

\section{Proportion of Engraved Fine wares}

Engraved fine ware sherds are ubiquitous in East Texas Caddo ceramic assemblages for a millennium, from ca. A.D. 850 to A.D. 1838. However, there are significant temporal and spatial differences in the relative proportions of engraved sherds in decorated sherd assemblages. Sites where engraved sherds comprise more than 40 percent of decorated sherd assemblages are found in the Red, Big Cypress, upper and middle Sabine, the upper Neches, and the lower Angelina River basin (Figure 2). These are both habitation and mound sites, although all the mound sites with high proportions of engraved sherds are located in the Red River basin: these include Eli Moores (41BW2), Hatchel (41BW3/41BW169), Fasken (41RR14), and Sanders (41LR2) (see Table 1).

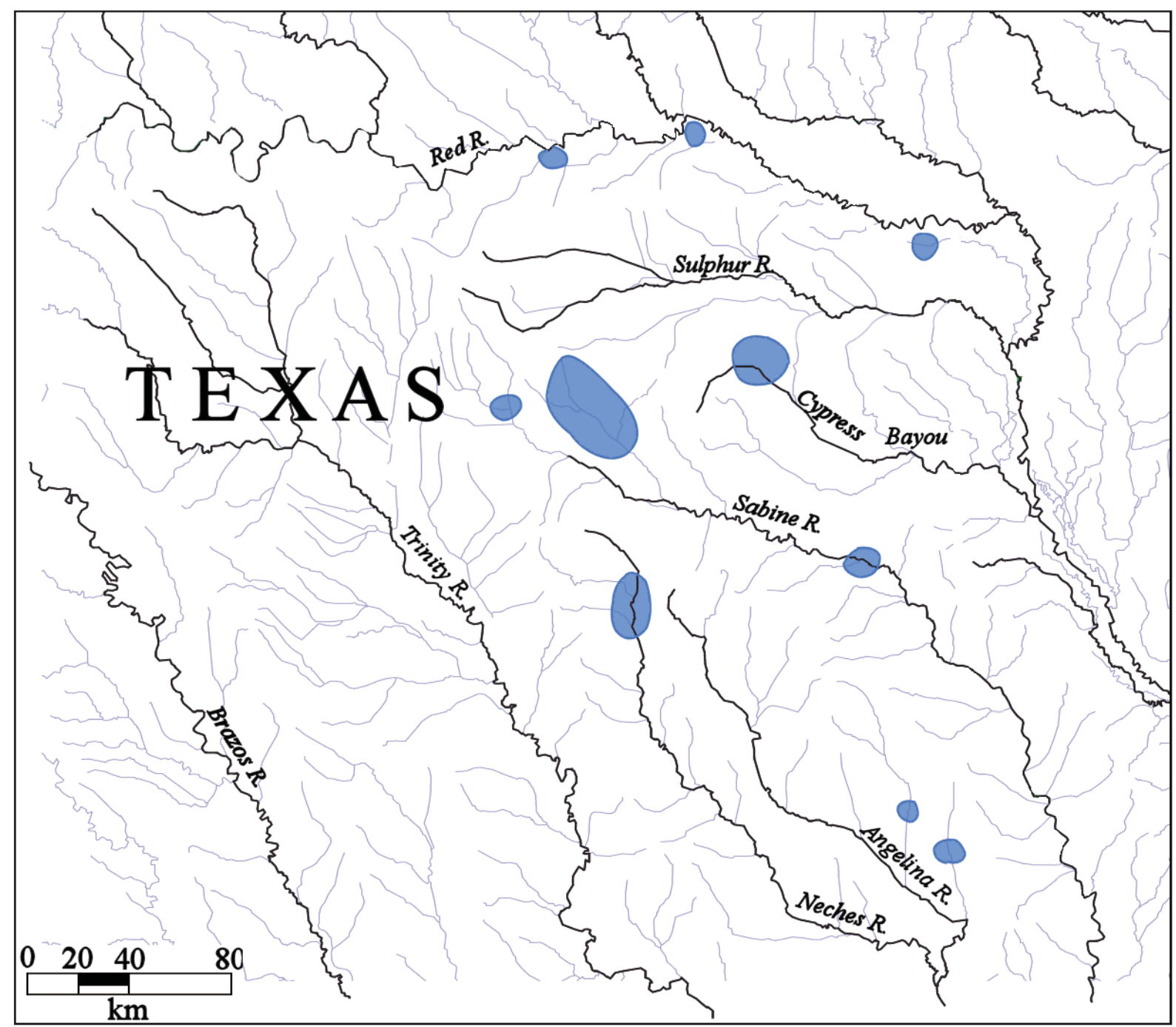

Figure 2. Areas with high proportions ( $>40$ percent of the decorated sherd assemblages) of engraved sherds in East Texas Caddo sites. 
Pre-A.D. 1400 sites $(n=8)$ with considerable amounts of engraved sherds in decorated sherd assemblages are present in the upper and middle Sabine, Big Cypress, and the middle reaches of the Red River (see Figure 2). Sites where engraved sherds comprise more than 40 percent of the decorated sherd assemblages are much more common $(\mathrm{n}=27)$ in ca. A.D. 1400-1830 Caddo sites throughout East Texas, particularly in Titus phase sites in the upper Sabine River and Big Cypress Creek basins, Frankston phase sites in the upper Neches, and Historic Caddo sites in the upper and middle Sabine, Angelina, and Red River basins (see Table 1).

\section{Use of Red-Slipped Ceramics}

Red-slipped fine wares (bowls, carinated bowls, and an occasional bottle) are a common part of ancestral Caddo ceramic assemblages in several parts of East Texas, most notably in sites in the middle Red River, the Big Cypress Creek basin, the upper Sulphur and Sabine River basins, and the middle Sabine River basin (Figure 3). The virtual absence of red-slipped sherds in ceramic assemblages from the Neches and Angelina River basins is particularly notable.

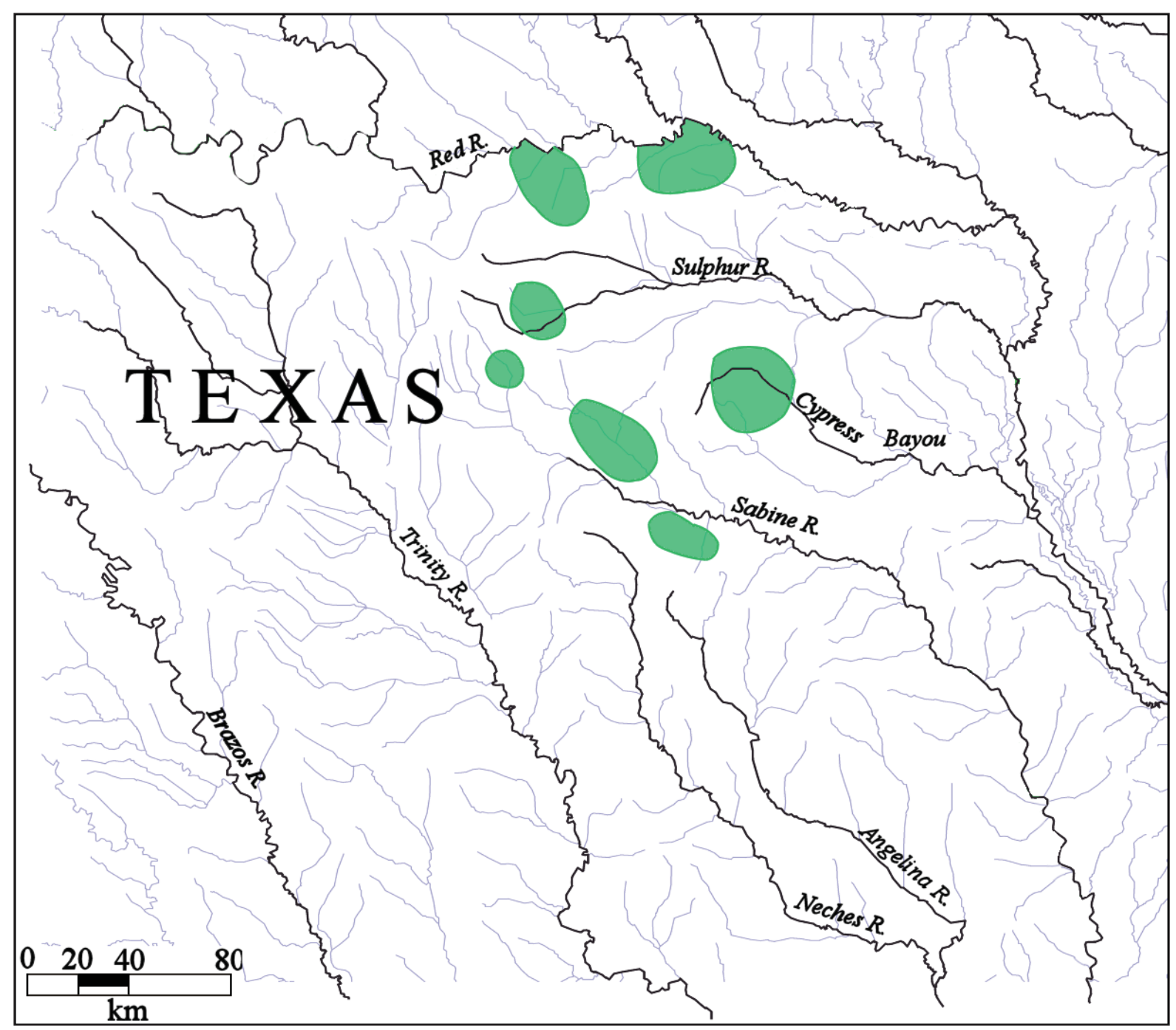

Figure 3. Caddo site clusters with high proportions ( $>10$ percent of decorated ceramic assemblages) of redslipped sherds in East Texas. 
Pre-A.D. 1400 ceramic assemblages where red-slipped sherds are relatively abundant are well represented in the aforementioned areas, particularly at sites such as Jamestown (41SM54), A. C. Gibson (41WD1), Sam Kaufman (41RR16), A. C. Mackin (41LR31), Harling (41FN1), and Sanders (41LR2) on the Sabine and Red rivers, respectively, several sites in the upper Sulphur River basin (41DT54 and 41DT63), and $41 \mathrm{TT} 110$ on Big Cypress Creek (see Table 1).

Later ceramic assemblages (i.e., dating after A.D. 1400) with red-slipped sherds are found in these same areas, most notably in shell-tempered wares (Clement Redware, see Flynn 1976) in McCurtain phase sites on the middle reaches of the Red River (see Figure 3) and the ca. A.D. 1680-1740 components at the Harling (41FN1) and Sanders sites. Other Late Caddo sites where red-slipped sherds are common in assemblages include Titus phase sites in the Big Cypress and upper Sabine River basins and 41HP175 in the upper Sulphur River basin (see Table 1).

\section{Trailed wares}

Sherds with trailed decorative elements, likely from Keno Trailed bowls and bottles (see Suhm and Jelks 1962), are found in percentages greater than 2 percent in ceramic assemblages in only a few parts of East Texas, principally in sites on the Red River (Figure 4). These sites generally date between ca. A.D. 1400 (or later) and A.D. 1730. The highest proportion of trailed sherds in ceramic assemblages (7.3-30.8 percent) are found in various Texarkana phase village and mound areas at the Hatchel site (41BW3) on the Red River (Perttula 2014b).

\section{Use of Brushed Ceramics}

Sherds from brushed utility ware vessels, particularly jars, are a distinctive characteristic of both Middle, Late, and Historic Caddo sites in much of East Texas. It also appears to be the case that the relative proportions of brushed utility wares increase through time in those areas where brushed vessels were made and used, such that sherds with brushing marks may comprise as much as 90 percent of all the decorated sherds in some post-A.D. 1400 East Texas ceramic assemblages.

In the East Texas Caddo ceramic sherd database, only a few ca. A.D. 1200-1430 sites have assemblages with high proportions ( $>60$ percent of the decorated sherd assemblage) of brushed sherds; these occur in the mid-Sabine and Big Cypress Creek drainage basins (see Table 1). Late Caddo ceramic assemblages in East Texas with high proportions of brushed sherds occur in the upper and mid-Neches (Frankston phase sites), Angelina, middle Sabine and Big Cypress (Titus phase sites), and sites (of unknown cultural taxonomy) on tributaries of the Sabine River west of the Toledo Bend Reservoir area (Figure 5). Caddo ceramic assemblages without considerable amounts of brushed sherds occur in the upper Sabine, Sulphur, and Red River basins.

Historic Caddo sites with high proportions of brushed sherds in ceramic assemblages are found principally in four parts of East Texas (see Figure 5). The first is in Allen phase sites $(n=15)$ in the upper Neches River basin (there is also one mid-Neches River basin Historic Caddo site, 41HO91, with abundant brushed sherds), A.D. 1700-1730 Nasoni Caddo sites $(n=3)$ in the western part of the Angelina River basin (Perttula et al. 2009), and other Allen phase sites/assemblages $(n=18)$ in the central part of the Angelina River basin.

One Historic Caddo Kinsloe phase site in the middle Sabine (41RK36) also has high proportions of brushed sherds in its decorated sherd assemblage (see Table 1). 


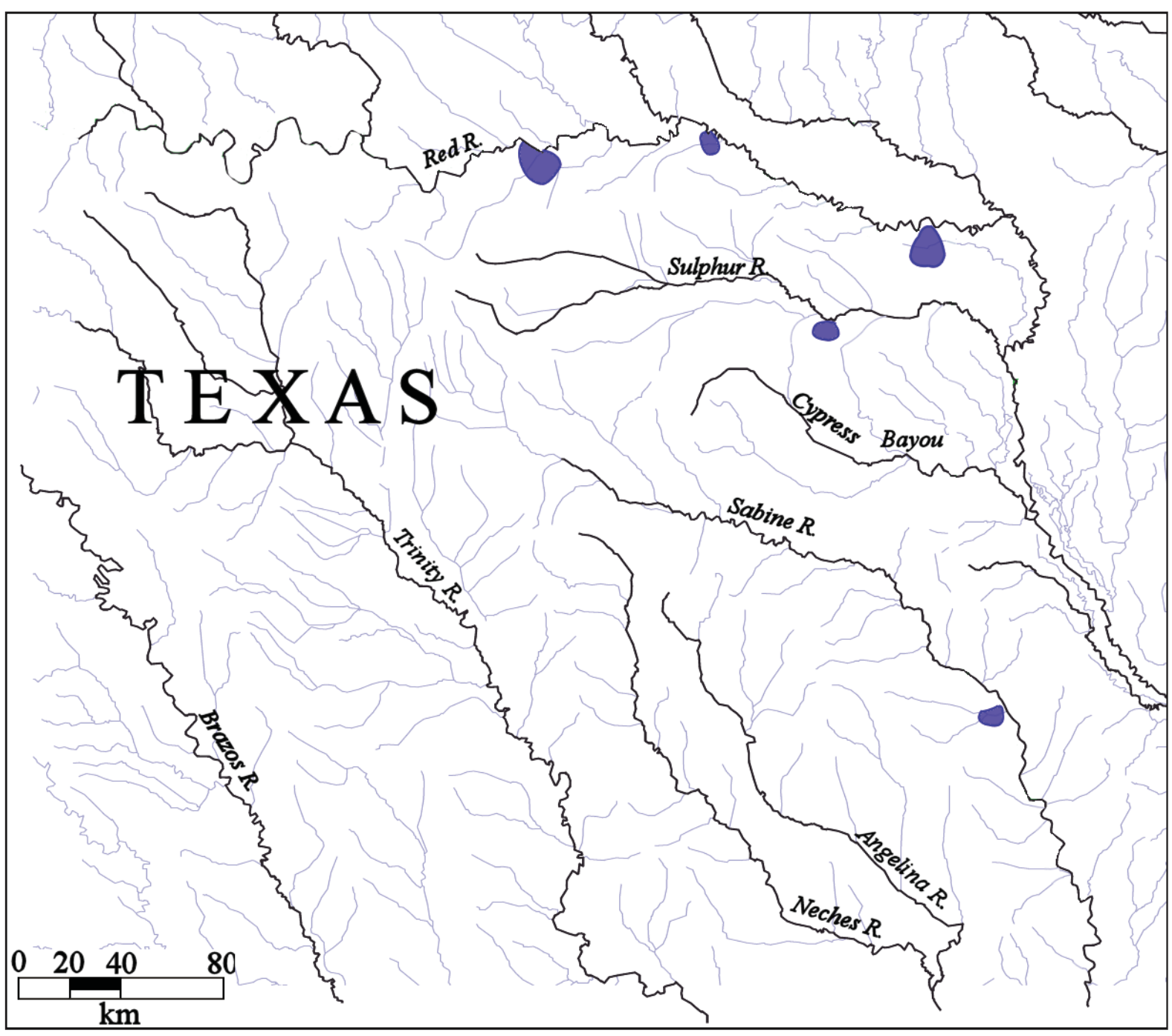

Figure 4. Distribution of sites where trailed sherds are most common in East Texas Caddo ceramic assemblages.

\section{Ridged Ceramics}

Ridged utility ware sherds (i.e., from Belcher Ridged jars, see Suhm and Jelks 1962:11 and Plate 6) are common (in proportions greater than 4.0 percent of decorated sherd assemblages) only in post-A.D. 1500 East Texas and western Louisiana Caddo communities in one locale along the Sabine River in the Toledo Bend Reservoir area (Figure 6). These are sites of undefined taxonomic affiliation, but they apparently are representative of "a local group whose ceramic tradition was distinct from Titus [phase] or Belcher [phase] in a number of ways. Certainly they had contacts with both these regions" (Kelley et al. 2010:26).

Belcher Ridged is one of the principal utility wares in Belcher phase sites on the Red River in northwestern Louisiana and southwestern Arkansas (see Figure 6). This area is more than ca. $70 \mathrm{~km}$ north of the Sabine River sites where ridged pottery is relatively common. In Titus phase sites on the middle Sabine and in the Big Cypress Creek basin - west of Belcher phase communities and ca. $70 \mathrm{~km}$ or more northwest of the Toledo Bend Reservoir Caddo communities with ridged pottery-only between 0.2-2.2 percent of the decorated sherds in their ceramic assemblages are from ridged jars. It is suspected that these sherds are from vessels made either by Belcher phase or the aforementioned middle Sabine Caddo potters. 


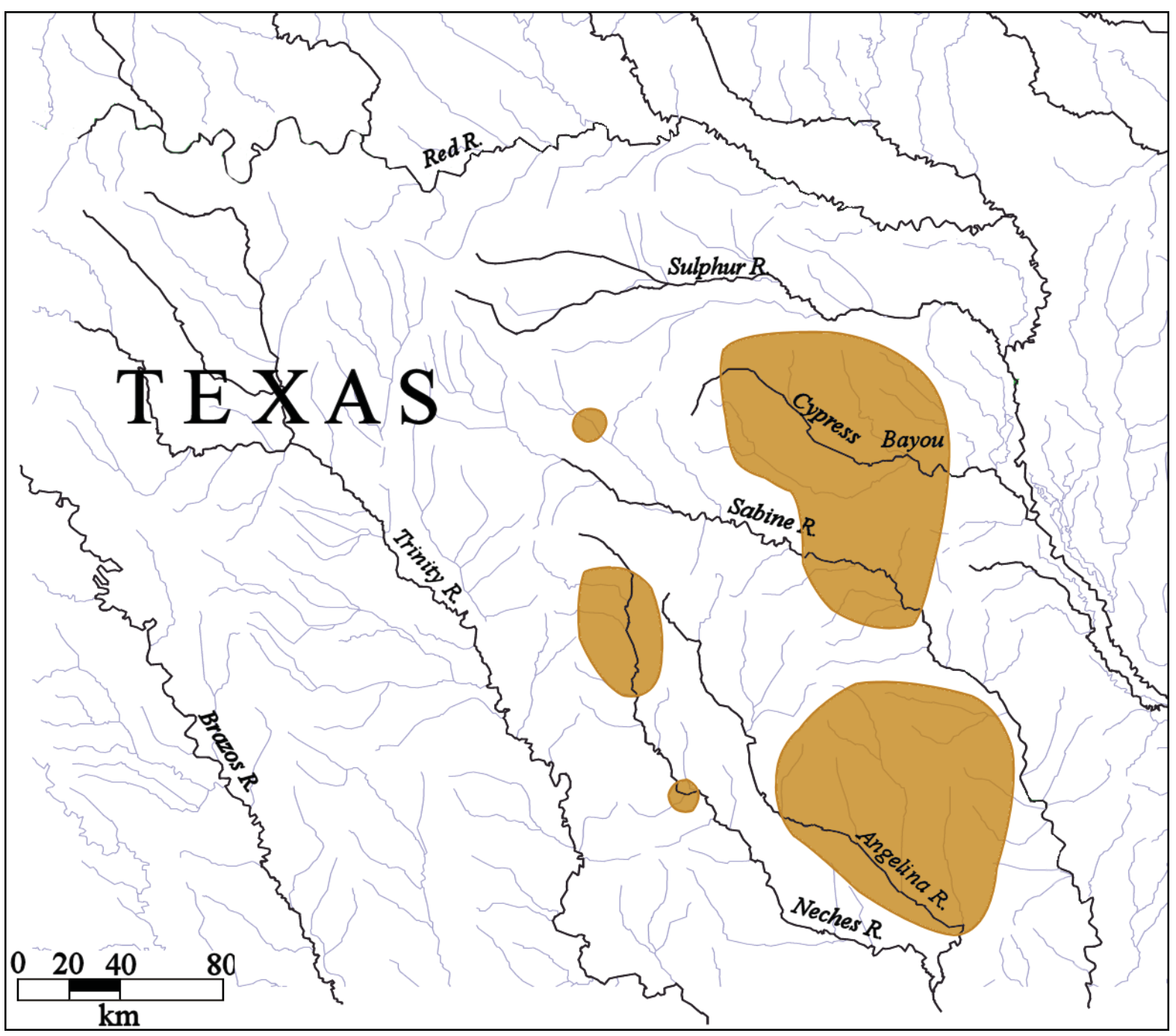

Figure 5. Caddo site clusters with high proportions ( $>60$ percent of decorated ceramic assemblages) of brushed sherds in East Texas.

\section{Other Utility Wares}

\section{Corn cob impressed}

Corn cob impressed sherds (i.e., Anglin Corn Cob Impressed) have been identified in only five sites in the East Texas Caddo ceramic sherd database (see Table 1). These sites occur only in the upper Sabine, Sulphur, and Red River basins in the region (Figure 7). In three of the sites, the corn cob impressed sherds date after ca. A.D. 1550, while in the two other sites the corn cob impressed sherds are in ca. A.D. 12001400 ceramic assemblages.

\section{Grooved}

Utility ware jar sherds with grooved decorative elements (i.e., from Lindsey Grooved vessels, see Marceaux 2011) are distributed in two clusters of Caddo sites in the upper Neches and Angelina river basins (Figure 8). These sites all date after ca. A.D. 1680 to ca. A.D. 1750 and are historic Caddo sites associated 


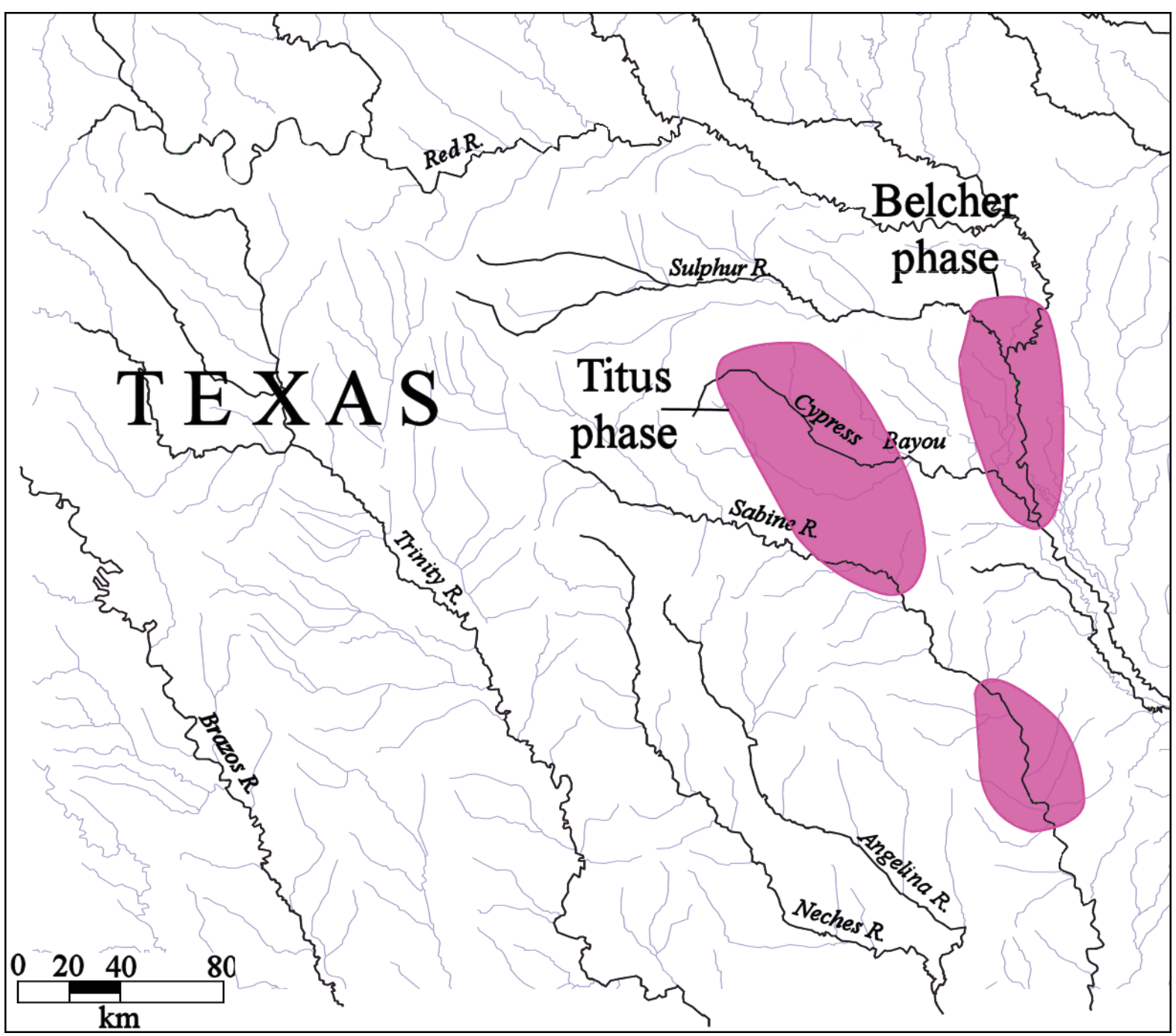

Figure 6. Distribution of sites where ridged ceramic sherds are present in East Texas, as well as the location of the Belcher phase and Titus phase sites with ridged ceramic sherds.

with the Allen phase (see Table 1). One grooved sherd from the Gilbert site (41RA13) in the upper Sabine River basin likely represents part of a vessel that was manufactured in one or the other of the two identified spatial clusters.

There are also a few grooved sherds from ca. A.D. 900-1300 contexts at three sites in the Neches, Red, and Sabine River basins, most notably at the George C. Davis site (41CE19). These grooved sherds are not related either stylistically or temporally with Lindsey Grooved wares, and are likely from Crenshaw Fluted vessels with deep vertical grooves or flutes (see Perttula and Selden 2015).

\section{Lip Notched}

The notching of the lips of vessels at the sole rim decoration is an apparently distinctive decorative method in a number of different Caddo communities of different ages in East Texas. The earliest assemblages $(\mathrm{n}=6)$, dating from ca. A.D. 900-1300, with lip notched vessels occur in the upper Red, upper and middle Sabine, and in the Angelina River basins (Figure 9). 


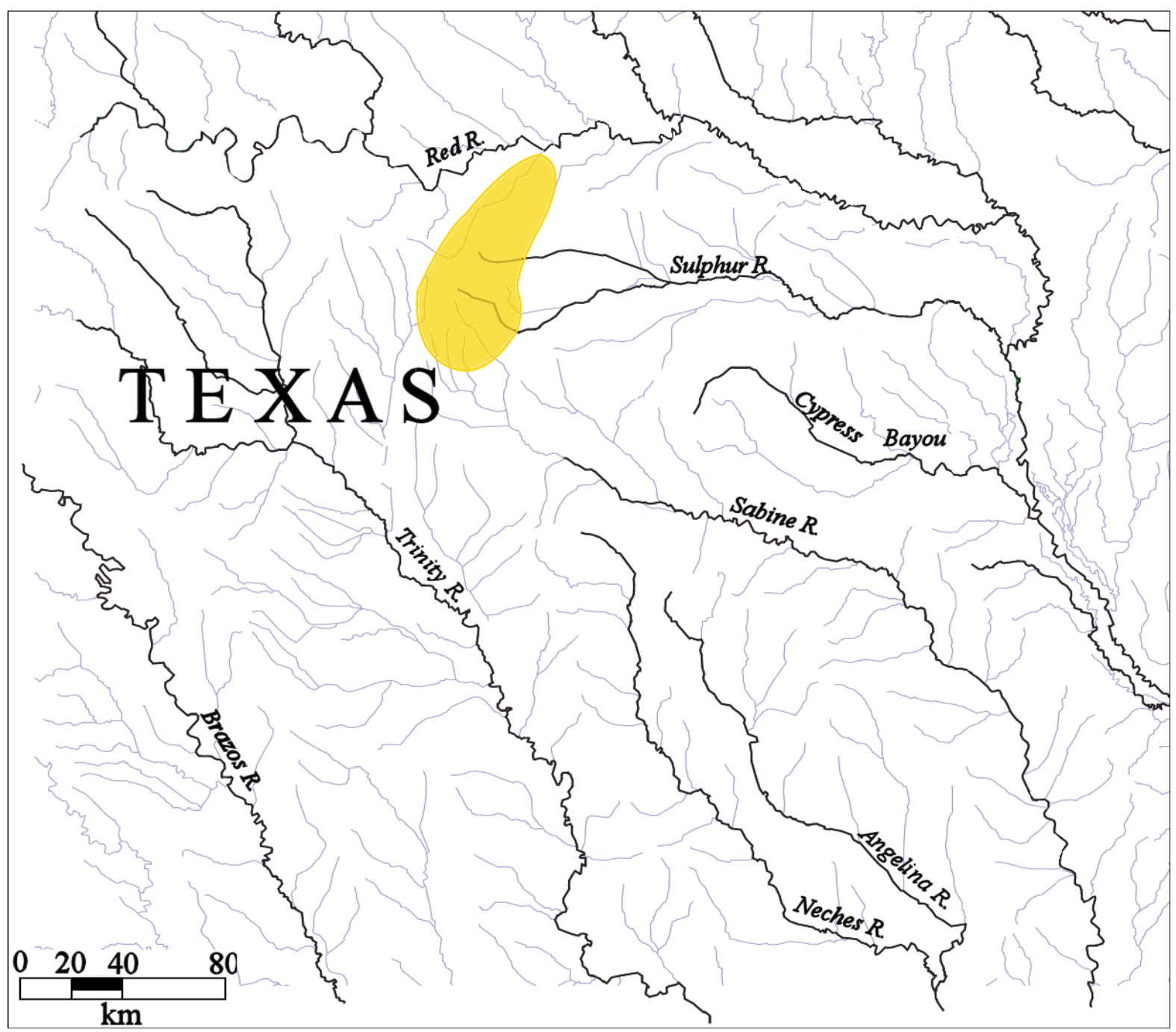

Figure 7. Distribution of sites with corn cob impressed sherds in East Texas.

Middle Caddo period communities where lip notched ceramics were made and eventually discarded $(\mathrm{n}=7)$ include the same previously mentioned assemblages, as well as sites in the Big Cypress Creek basin (see Figure 9). By post-A.D. 1400 times until the early $18^{\text {th }}$ century, ceramic assemblages with lip notched vessels $(\mathrm{n}=10)$ occur more regularly in the upper Neches, middle Red River, middle Sabine, and the Angelina River basin (see Figure 9).

\section{Neck Banded}

Neck banded jars were a common utility ware in a number of ancestral Caddo communities occupied after ca. A.D. 1300 in East Texas (Figure 10), including both grog-bone and shell-tempered varieties. The highest proportions (23.6-79.6 percent of the decorated sherd assemblage) of neck banded sherds (shell-tempered) occur in ca. A.D. 1400-1680 McCurtain phase assemblages on the middle reaches of the Red River. Shell-tempered neck banded sherds (Nash Neck Banded) are also found in high percentages at other sites on the same age in other Red River communities (Figure 10) both upstream and downstream from the McCurtain phase sites; both grog/bone and shell-tempered neck banded sherds are found in these areas. 


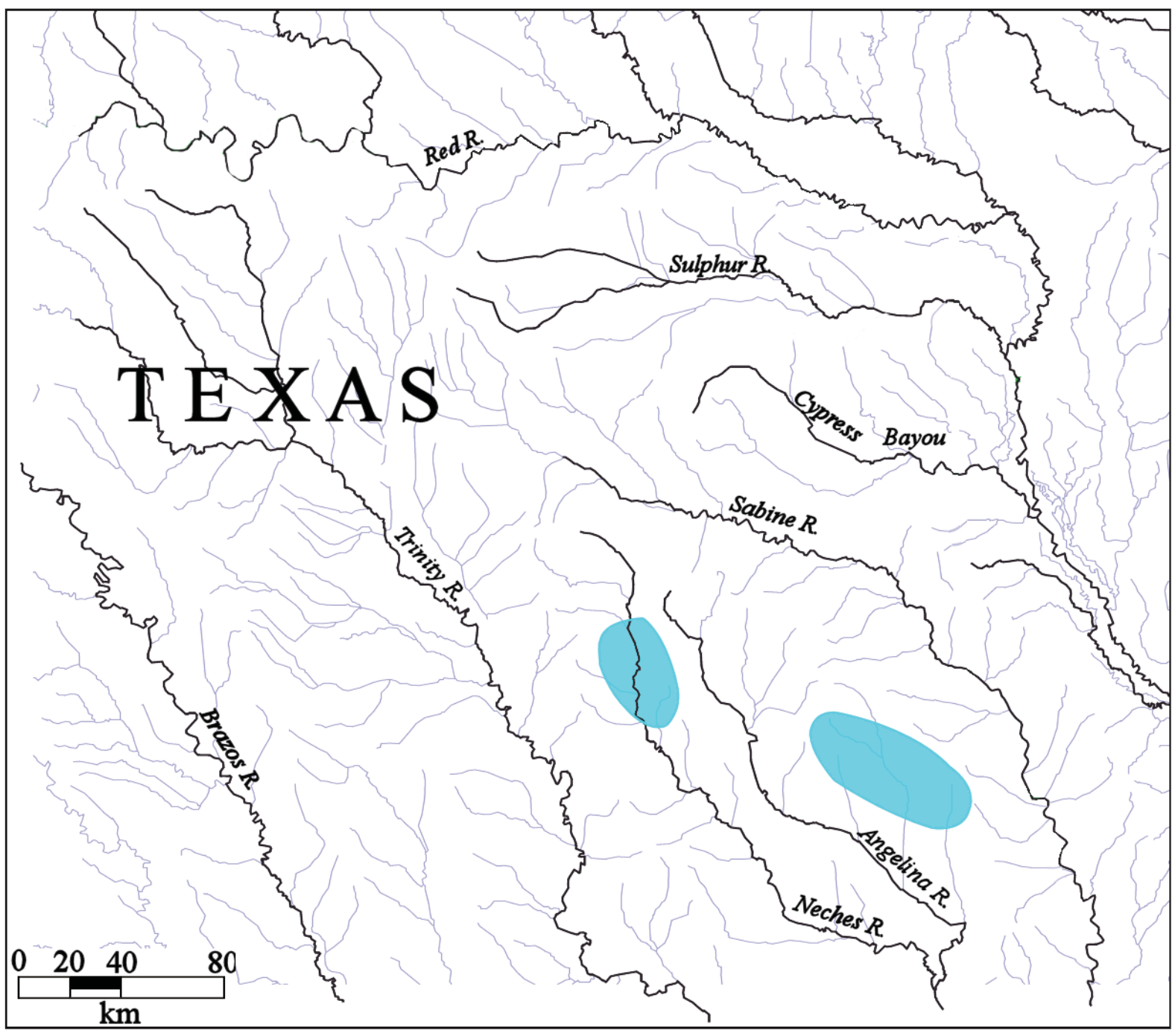

Figure 8. Distribution of East Texas Caddo sites with grooved sherds.

In other locales across East Texas, neck banded wares are almost exclusively grog/bone-tempered. These wares are found in sites in the upper Neches and upper Sabine River basins in Frankston (ca. A.D. 1400-1650) and Titus phase (ca. A.D. 1430-1680) contexts and in Titus phase ceramic assemblages in the Big Cypress Creek basin (see Figure 10). This ware has been classified as La Rue Neck Banded.

\section{Spatial and Temporal Differences in Temper Use}

The principal tempering materials used by East Texas Caddo potters from as early as ca. A.D. 850 were grog (crushed sherds) and burned bone. The use of grog temper occurs in East Texas Caddo assemblages in each of the river basins, irrespective of their age, but the common use of burned bone has distinct spatial and temporal distributions. So too does the use of burned mussel shell by Caddo potters, although its use is much more restrictive temporally and spatially than is burned bone (see Table 1). 


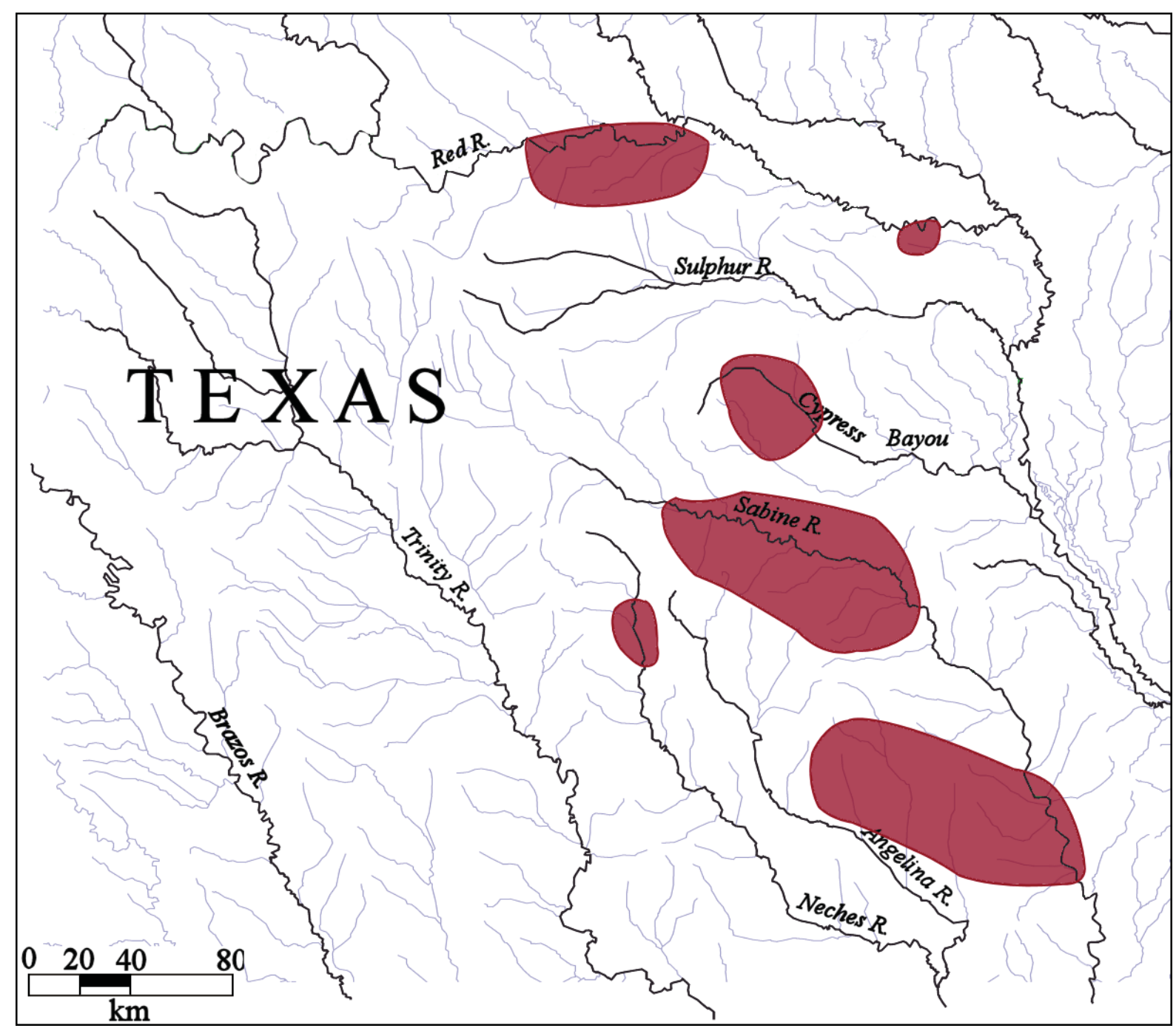

Figure 9. Distribution of Caddo site clusters with lip notched sherds in East Texas.

\section{Bone-tempered ceramics}

The use of burned animal bone for the temper of ceramic vessels is a distinctive characteristic of East Texas Caddo ceramic sherd assemblages, and most ceramic assemblages in the region have some bonetempered sherds (see Table 1). However, sherd assemblages with high proportions ( $>40$ percent of the sherd assemblage) of bone temper are concentrated in only a few locales across East Texas, most notably in the Toledo Bend Reservoir area along the middle Sabine River and in sites in the Angelina River basin (Figure 11). Bone-tempered sherds are not a notable feature of Caddo ceramic assemblages in the Neches, Big Cypress, Sulphur, or Red River basins.

Pre-A.D. 1400/1450 Caddo sites with a high proportion of bone temper are found only in a few areas in the middle Sabine River basin, including the Redwine site (41SM193) (see Table 1). Late Caddo (ca. A.D. 1400-1680) sites and assemblages with high proportions of bone temper are found in one site in the Trinity River basin (41HE70, Story 1965), and in several sites in the mid-Sabine and Angelina River basins (see Figure 11). In fact, these sites are part of a previously identified Late Caddo bone-tempered and brushed ceramic tradition (Perttula et al. 2011b:Figure 6-71). Historic Caddo sites (dating from ca. A.D. 1700-1830) with high percentages of bone temper use are known only in the upper and middle Sabine River basins, and in the lower Angelina River basin (see Figure 11). 


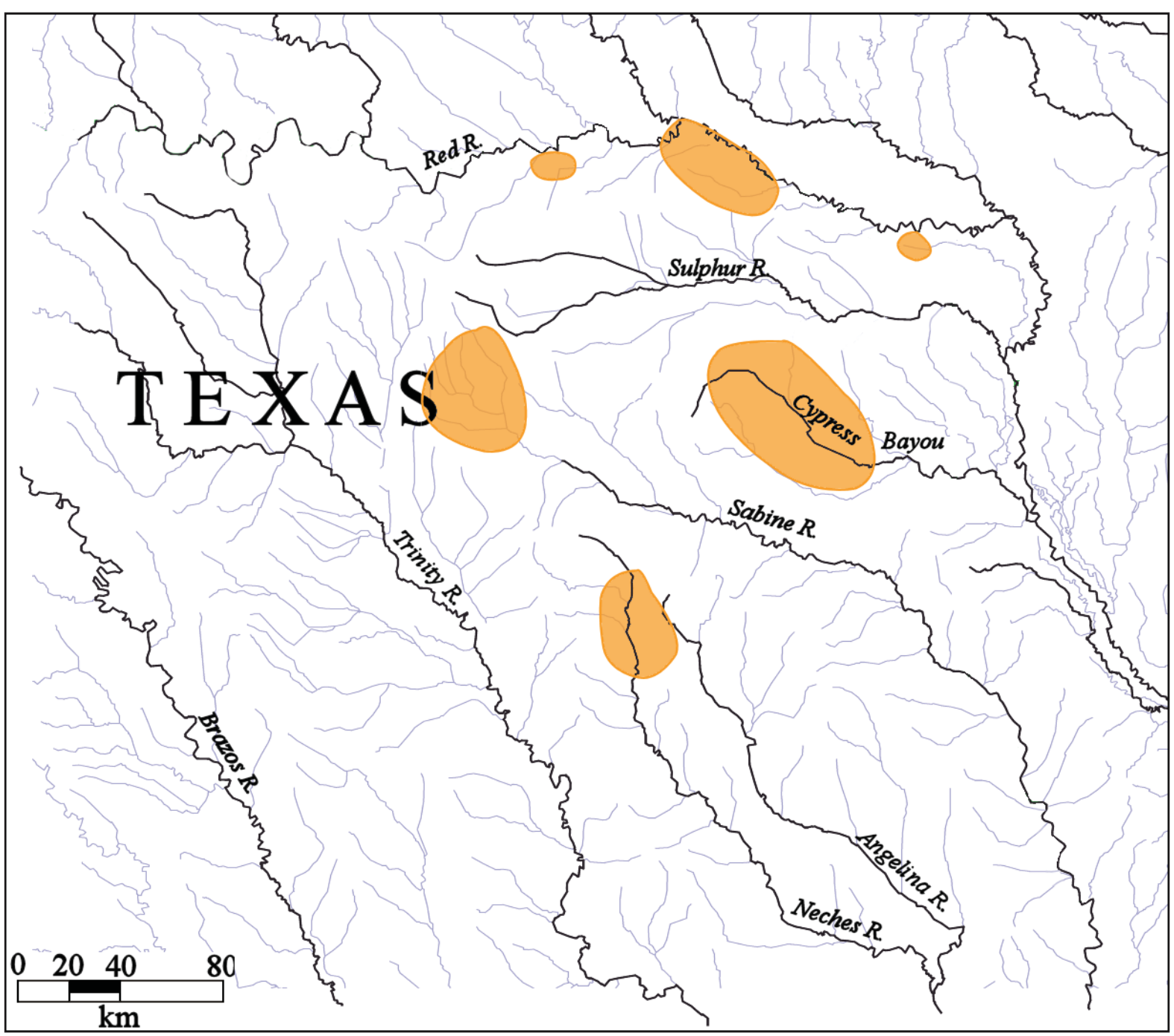

Figure 10. Distribution of areas of Caddo sites with high proportions ( $>5$ percent of the decorated sherd assemblage) of neck banded sherds in East Texas.

\section{Shell-tempered ceramics}

Perttula et al. (2012e) have reviewed the age and distribution of shell-tempered ceramics in Caddo sites across the southern Caddo area. In general, shell-tempered ceramics were made by East Texas Caddo potters after ca. A.D. 1300, particularly in McCurtain phase sites along the middle reaches of the Red River and the lower Kiamichi River in southeastern Oklahoma (Figure 12; see also Selden et al. 2014:Figure 4). In East Texas McCurtain phase ceramic assemblages, the proportions of shell-tempered sherds ranges from 93-100 percent (see Table 1).

Caddo sites dating between ca. A.D. 1400-1680 with considerable amounts of shell-tempered ceramic sherds are foud at just a few sites in the upper Sulphur and the mid-Red River (41BW716) (see Figure 12). By contrast, high proportions of shell-tempered sherds in ceramic assemblages are relatively common in post-A.D. 1700 Historic Caddo sites only in the upper Sabine, the Big Cypress Creek basin, and in two locales on the Red River, both upstream and downstream from the McCurtain phase sites (see Figure 12 and Table 1). 


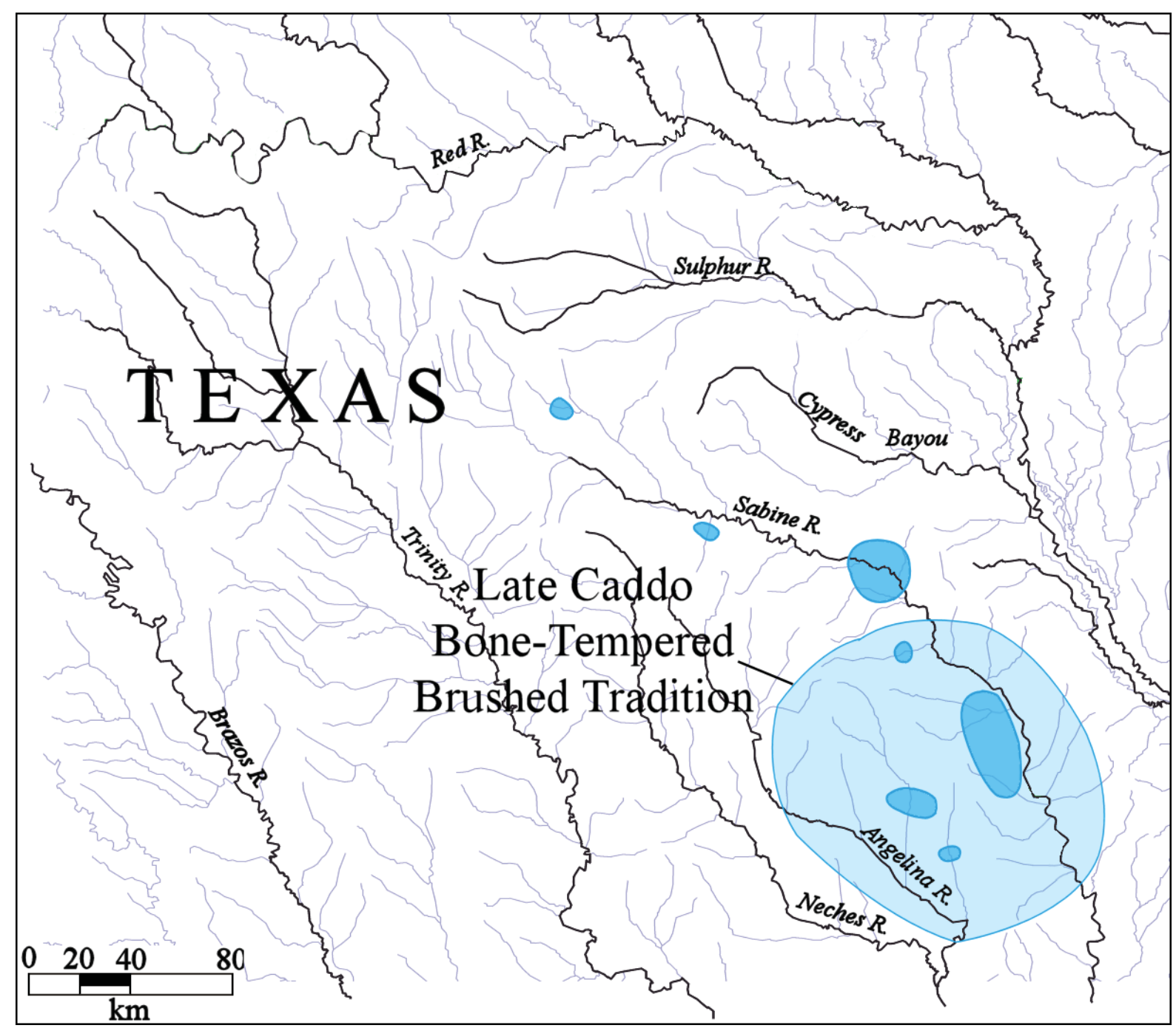

Figure 11. Clusters of Caddo sites with high proportions (>than 40 percent) of bone-tempered sherds in East Texas ceramic assemblages.

\section{FUTURE PROSPECTS}

The analytical and archaeological findings reported on in this article are based on a consideration of the East Texas Caddo ceramic sherd database, and represent only an initial set of stylistic attributes that have distinctive spatial and temporal distributions across East Texas. These findings barely plumb the depths of the East Texas Caddo ceramic sherd database, and further analyses are warranted; hopefully other ceramic assemblages can also be added to the database.

The next step will be to more formally and statistically assess the regional variation in Caddo ceramic assemblages. This should be based on a further delineation of temporal (i.e., to the smallest temporal interval possible given available chronological data) and spatial divisions in the character of Caddo ceramics (i.e., principally data on decorative methods and the use of different tempers) across East Texas sites, and then constructing networks of similarities between ceramic assemblages from these sites (cf. Peeples and Roberts 2013:3003-3004) that can be used to assess the strength of cultural relationships among Caddo communities in the region through time and across space. These postulated relationships should then be explored to try to determine the underlying reasons for the existence of such relationships, including factors such as the 


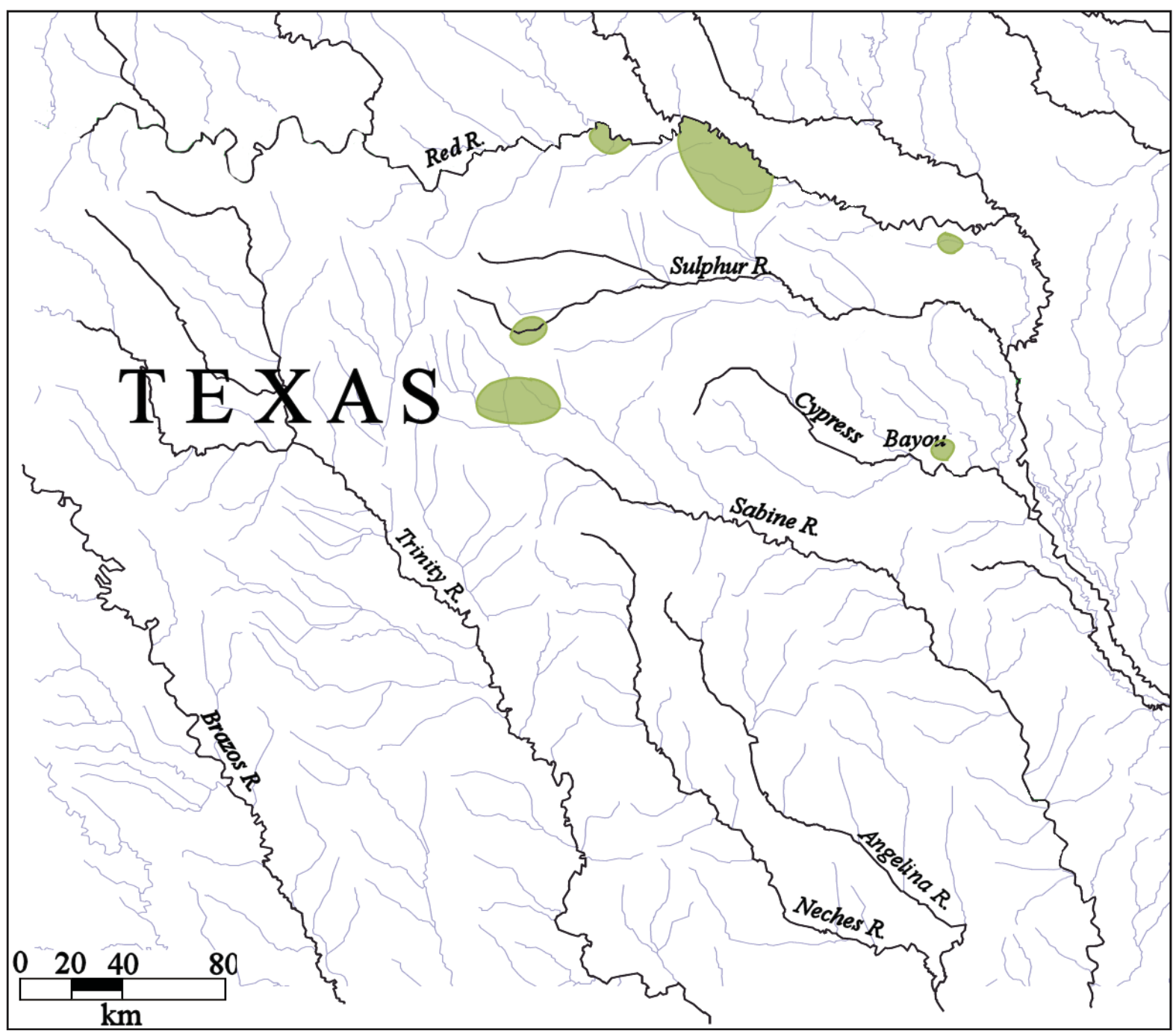

Figure 12. Clusters of Caddo sites with high proportions of shell-tempered sherds in East Texas ceramic assemblages.

frequency of interaction and direct contact between communities, trade and exchange of ceramic vessels, population movement, and similarities in the organization of ceramic vessel production. The results of past and current instrumental neutron activation analysis (INAA) and petrographic analysis of Caddo Area ceramics, including East Texas (where there is a robust INAA database) should also be explored as a means to corroborate production locales (cf. Selden et al. 2014), establish the chemical and paste characteristics of local fine ware and utility ware ceramics in assemblages, and evaluate the possible movement of ceramic vessels between different Caddo communities in East Texas and the broader Caddo world.

Finally, in conjunction with a database on 2D/3D-scanned Caddo ceramic vessels from East Texas sites, the East Texas Caddo ceramic sherd database should be made part of a digital database where comprehensive mathematical and quantitative analyses of morphological attributes and decorative elements on sherds and vessels can be conducted (e.g., Smith et al. 2014). Queries to such a combined database of vessels and sherds should lead to better understandings of regional Caddo ceramic typologies and their spatial and temporal underpinnings. 


\section{REFERENCES CITED}

Anderson, K. M., K. Gilmore, O. F. McCormick III, and E. P. Morenon

1974 Archaeological Investigations at Lake Palestine, Texas. Contributions in Anthropology No. 11. Department of Anthropology, Southern Methodist University, Dallas.

Barnhart, E., B. Dixon, S. Kotter, M. Nash, K. Reese-Taylor, E. Skokan, and R. Taylor

1997 Data Recovery Excavations at Site 41 TT372 and 41 TT550 in the Tankersley and Hayes Creek Watersheds, Monticello B-2 Surface Mine, Titus County, Texas. Document No. 940608. Espey Huston \& Associates, Inc., Austin.

Bell, M.

1981 The Alex Justiss Site. A Caddoan Cemetery in Titus County, Texas. Publications in Archaeology No. 21. Highway Design Division, Texas Department of Highways and Public Transportation, Austin.

Benham, B. L., H. L. Miller, and J. V Sciscenti

1973 Archaeological Research in the Toledo Bend Reservoir. Archaeology Research Program, Southern Methodist University, Dallas.

Brewington, R. L., J. E. Dockall, and H. J. Shafer

1995 Archaeology of 41MX5: A Late Prehistoric Caddoan Hamlet in Morris County, Texas. Reports of Investigations No. 1. Center for Environmental Archaeology, Texas A\&M University, College Station.

Bruseth, J. E. and T. K. Perttula

1980 Archaeological Research at Lake Fork Reservoir: Excavations at the Howle Site and Site Testing. Archaeology Research Program, Southern Methodist University, Dallas.

1981 Prehistoric Settlement Patterns at Lake Fork Reservoir. Texas Antiquities Permit Series, Report No. 2. Texas Antiquities Committee and Southern Methodist University, Austin and Dallas.

2006 Archeological Investigations at the Hudnall-Pirtle Site (41RK4): An Early Caddo Mound Center in Northeast Texas. Caddo Archeological Journal 15:57-158.

Campbell, J. A.

2001 Addendum I to Phase I Archaeological Investigations for the Proposed Longview Transmission Line Project, Harrison, Upshur, and Gregg Counties. Burns \& McDonnell, Kansas City.

Cliff, M. B. and T. K. Perttula

2002 Results of National Register Investigations Conducted on Site 41PN175, Panola County, Texas. Report No. 32. Archeological Studies Program, Environmental Affairs Division, Texas Department of Transportation, Austin.

Cliff, M. B., S. M. Hunt, M. M. Green, R. Proctor, F. B. Largent, Jr., and W. J. Autin

1997 Geomorphological Investigations and Inventory of Cultural Resources along and near the Bowie County Levee, Bowie County, Texas: 1996. Miscellaneous Report of Investigations No. 139. Geo-Marine, Inc., Plano.

Cliff, M. B., E. C. Sills, T. K. Perttula, and P. Dering

2004 National Register Testing of Sites 41HE14, 41HE139, and 41HE343 within Proposed FM 3506 Right of Way, Henderson County, Texas. Report No. 60. Archeological Studies Program, Texas Department of Transportation, Austin.

Clark, J. W. and J. E. Ivey

1974 Archaeological and Historical Investigations at Martin Lake, Rusk and Panola Counties, Texas. Research Report 32. Texas Archeological Survey, Austin. 
Corbin, J. E. and D. C. Kisling

1983 The Adolphus Sterne Home: Preliminary Archaeological Investigations of a Mid-Nineteenth Century Plantation in Nacogdoches County, Texas. Papers in Anthropology No. 4. Stephen F. Austin State University, Nacogdoches.

Corbin, J. E., H. A. Brown, M. G. Canavan, and S. Toups

1990 Mission Dolores de los Ais (41SA25): San Augustine County Texas. Archeological Investigations, Stephen F. Austin State University, Nacogdoches

Creel, D. G.

1979 Archeological Investigations at the George C. Davis Site, Cherokee County, Texas, Summer 1978. Texas Antiquities Permit Series No. 1. Texas A\&M University, College Station.

Davis, E. M., W. A. Davis, J. R. Gipson, and B. Golden

2010 Archeological Investigations at Lake O'The Pines, Marion and Upshur Counties, Texas, 1957-1959. Archival Series 4. Texas Archeological Research Laboratory, The University of Texas at Austin.

Davis, W. A. and H. R. Horn

1964 The Zavonian Springs Site: An Archaic-Neo-American Site in McGee Bend Reservoir, San Augustine County, Texas. Bulletin of the Texas Archeological Society 35:113-150.

Dixon, B., S. Kotter, E. Skokan, M. Nash, R. Rogers, and E. Barnhart

1995 Archaeological Testing of Site 41 TT672 and Geomorphological Exploration of Tankersley and Dragoo Creek Drainages, Titus County, Texas. Document No. 950565. Espey, Huston \& Associates, Inc., Austin.

Dockall, J. E. and R. C. Fields

2011 National Register Testing of Three Sites in the Sabine Mine's South Hallsville No. 1 Mine-Rusk Permit, Rusk County, Texas. Report of Investigations No. 162. Prewitt and Associates, Inc., Austin.

Dockall, J., S. Katauskas, and R. Fields

2008 National Register Testing of Four Sites in the Sabine Mine's Area M, Harrison County, Texas. Reports of Investigations No. 157. Prewitt and Associates, Inc., Austin.

Doehner, K. and R. E. Larson

1978 Archaeological Research at Cooper Lake, Northeast Texas, 1974-75. Research Report No. 108. Archaeology Research Program, Southern Methodist University, Dallas.

Doehner, K., D. Peter, and S. A. Skinner

1978 Evaluation of the Archaeology at the Proposed Cooper Lake. Research Report No. 114. Archaeology Research Program, Southern Methodist University, Dallas.

Duffield, L. F.

1961 The Limerick Site at Iron Bridge Reservoir, Rains County, Texas. Bulletin of the Texas Archeological Society 30:51-116.

Duffield, L. F. and E. B. Jelks

1961 The Pearson Site: A Historic Indian Site at Iron Bridge Reservoir, Rains County, Texas. Archaeology Series No. 4. Department of Anthropology, The University of Texas at Austin.

Fields, R. C.

1978 Report on the 1977 Investigations at the George C. Davis Site, Caddoan Mounds State Historic Site, Cherokee County, Texas. Texas Archeological Research Laboratory, The University of Texas at Austin.

1995 Analysis of Native-Made Ceramics. In The Deshazo Site, Nacogdoches County. Texas, Volume 2: Artifacts of Native Manufacture, edited by D. A. Story, pp. 173-232. Studies in Archeology 21. Texas Archeological Research Laboratory The University of Texas at Austin. 
Fields, R. C. and E. F. Gadus (editors)

2012 Archeology of the Nadaco Caddo: The View from the Pine Tree Mound Site (41HS15), Harrison County, Texas. 2 Vols. Reports of Investigations No. 164. Prewitt and Associates, Inc., Austin.

Fields, R. C. and J. P. Thurmond

1980 The George C. Davis Site, Cherokee County, Texas: Spring 1980 Archeological Investigations. Report of Investigations No. 8. Prewitt and Associates, Inc., Austin.

Fields, R. C., E. F. Gadus, and L. W. Klement

1994a The Peerless Bottoms Site: A Late Caddoan Component at Cooper Lake, Hopkins County, Texas. Bulletin of the Texas Archeological Society 65:55-114.

Fields, R. C., E. F. Gadus, L. W. Klement, and K. M. Gardner

1994b Excavations at the Spider Knoll Site, Cooper Lake Project, Delta County, Texas. Reports of Investigations No. 96. Prewitt and Associates, Inc., Austin.

Fields, R. C., E. F. Gadus, L. W. Klement, C. B. Bousman, and J. B. McLerran

1993 Excavations at the Tick, Spike, Johns Creek, and Peerless Bottoms Sites, Cooper Lake Project, Delta \& Hopkins Counties, Texas. Report of Investigations No. 91. Prewitt and Associates, Inc., Austin.

Fields, R. C., V. L. Hatfield, D. Burden, E. F. Gadus, M. C. Wilder, and K. W. Kibler

2014 Testing and Data recovery Excavations at 11 Native American Archeological Sites along the U.S. Highway 271 Mount Pleasant Relief Route, Titus County, Texas. 2 Vols. Reports of Investigations No. 168. Prewitt and Associates, Inc., Austin.

Flynn, P.

1976 A Study of Red-Filmed Pottery from the Clement Site (Mc-8), McCurtain County, Oklahoma. Bulletin of the Oklahoma Anthropological Society 25:127-134.

Gadus, E. F., R. C. Fields, and C. B. Bousman

1992 Archeological Investigations at 41DT11, 41DT21, 41DT50, 41DT54, and 41DT63 at Cooper Lake, Delta County, Texas. Reports of Investigations No. 86. Prewitt and Associates, Inc., Austin.

Gadus, E. F., R. C. Fields, J. K. McWilliams, J. Dockall, and M. C. Wilder

2006 National Register Testing of Seven Prehistoric Sites in the Sabine Mine's Area Q, Harrison County, Texas. Reports of Investigations, Number 147. Prewitt and Associates, Inc., Austin.

Gadus, E. F., J. K. McWilliams, and R. C. Fields

2002 Data Recovery Excavations at the McGuire's Garden Site (41FT425), Jewett Mine, Freestone County, Texas. Reports of Investigations No. 134. Prewitt and Associates, Inc., Austin.

Galan, V., R. Rogers, T. K. Perttula, and E. S. Switek

1997 National Register Testing of Seven Sites in the Monticello B-2 Surface Mine, Titus County, Texas. Document No. 971085 . Espey, Huston \& Associates, Inc., Austin.

Gilmore, K.

1986 French-Indian Interaction at an Early Eighteenth Century Post: The Roseborough Lake Site, Bowie County, Texas. Contributions in Archaeology 3. Institute of Applied Sciences, North Texas State University, Denton.

Goode, G. T., T. K. Perttula, L. L. Bush, S. Marceaux, L. Schniebs, and J. Todd

2014 Excavations at the Early Caddo Period Mound Pond Site (41HS12) in Harrison County, Texas. MS on file, Center for Regional Heritage Research, Stephen F. Austin State University, Nacogdoches.

Griffith, T. B., R. C. Fields, S. L. Katauskas, and A. E. Dase

2012 Archeological and Historical Resources Surveys of 2,144 Acres in the Proposed Marshall Mine, Harrison and Panola Counties, Texas. Reports of Investigations No. 163. Prewitt and Associates, Inc., Austin. 
Harris, R. K., I. M. Harris, J. C. Blaine, and J. Blaine

1965 A Preliminary Archeological and Documentary Study of the Womack Site, Lamar County, Texas. Bulletin of the Texas Archeological Society 36:287-365.

Hart, J. P.

1982 An Analysis of the Aboriginal Ceramics from the Washington Square Mound Site, Nacogdoches County, Texas. Master's thesis, Department of Anthropology, Northeast Louisiana University, Monroe.

Haskins, P. and M. Walters

2001 Archaeological Investigations of an Oil Well Pad Disturbance at the Tom Moore Site (41PN149), Panola County. Journal of Northeast Texas Archaeology 14:37-61.

Heartfield, Price, and Greene, Inc.

1988 Data Recovery at 41HS74, Harrison County, Texas. Heartfield, Price, and Greene, Inc., Monroe.

Hunt, S. M., F. B. Largent, Jr., and M. B. Cliff

1996 Cultural Resources Evaluation of the Pilgrim's Pride Property South of Big Cypress Creek, Camp County, Texas. Miscellaneous Report of Investigations No. 118. Geo-Marine, Inc., Plano.

Hyatt, R. D. and K. Doehner

1975 Archaeological Research at Cooper Reservoir, Northeast Texas, 1973. Contributions in Anthropology No. 15. Department of Anthropology, Southern Methodist University, Dallas.

Hyatt, R. D., B. H. Butler, and H. P. Mosca, III

1974 Archaeological Research at Cooper Lake 1970-1972. Contributions in Anthropology No. 12. Department of Anthropology, Southern Methodist University, Dallas.

Jackson, M. K., T. Middlebrook, G. Avery, H. Shafer, and B. Meissner

2012 Trade and Cultural Interaction along El Camino Real de los Tejas During the Spanish Colonial and Republic Periods in Nacogdoches County, Texas. 2 Vols. Nine Flags Museum, Nacogdoches.

Jelks, E. B.

1965 The Archeology of McGee Bend Reservoir, Texas. Ph.D. dissertation, Department of Anthropology, The University of Texas at Austin.

Jelks, E. B. and C. D. Tunnell

1959 The Harroun Site, A Fulton Aspect Component of the Caddoan Area, Upshur County, Texas. Archaeology Series No. 2. Department of Anthropology, The University of Texas at Austin.

Johnson, L., Jr.

1961 An Archeological Survey of Blackburn Crossing Reservoir on the Upper Neches River. Bulletin of the Texas Archeological Society 31:213-238.

1962 The Yarbrough and Miller Sites of Northeastern Texas, with a Preliminary Definition of the LaHarpe Aspect. Bulletin of the Texas Archeological Society 32:141-284.

Jones, B. C.

1957 The Grace Creek Sites, Gregg County, Texas. Bulletin of the Texas Archeological Society 28:198-231.

1968 The Kinsloe Focus: A Study of Seven Historic Caddoan Sites in Northeast Texas. Master's thesis, Department of Anthropology, University of Oklahoma, Norman.

Jurney, D. H.

2000 Passport in Time Archaeological Investigations at the Hargrove Lake Site (41HO150), Houston County, Texas. U.S. Forest Service, Lufkin. 
Jurney, D. H., J. Bohlin, S. E. Linder Linsley, S. C. Caran, and D. R. Pedler

1993 Archaeological Survey of Cooper Lake, Delivery Order Number 7, 1989. Cultural Resources Studies for Cooper Lake, Hopkins and Delta Counties, Texas. Archaeology Research Program, Southern Methodist University, Dallas.

Kelley, D. B.

2006 The Burnitt Site: A Late Caddoan Occupation in the Uplands of the Sabine River Basin of Louisiana. Coastal Environments, Inc., Baton Rouge.

Kelley, D. B., D. G. Hunter, K. M. Roberts, S. L. Scott, and B. S. Haley

2010 The Burnitt Site (16SA204): A Late Caddoan Occupation in the Uplands of the Sabine River Basin. Louisiana Archaeology 31:4-33.

Kenmotsu, N. A.

2005 Investigations at the Salt Well Slough Site (41RR204), a Salt Making Site in Red River County, Texas. Archeological Reports Series, No. 4. Texas Historical Commission, Austin.

Kleinschmidt, U. K. W.

1982 Review and Analysis of the A. C. Saunders Site, 41AN19, Anderson County, Texas. Master's thesis, Department of Anthropology, The University of Texas at Austin.

Krieger, A. D.

1941 An Analytical System for East Texas Pottery. Southeastern Archaeological Conference Newsletter 2(4):79.

1946 Culture Complexes and Chronology in Northern Texas, with Extensions of Puebloan Datings to the Mississippi Valley. Publication No. 4640. The University of Texas, Austin.

2000 The Pottery of the Sanders Farm. In The 1931 Excavations at the Sanders Site, Lamar County, Texas: Notes on the Fieldwork, Human Osteology, and Ceramics, by A. T. Jackson, M. S. Goldstein, and A. D. Krieger, pp. 131-144. Archival Series 2. Texas Archeological Research Laboratory, The University of Texas at Austin.

Largent, F. B., Jr., D. Beene, M. B. Cliff, and S. Hunt

1997 Cultural Resources Testing of Two Sites within the White Oak Creek Wildife Management Area (WOCMA), Bowie and Titus Counties, Texas. White Oak Creek Wildlife Management Area Archaeological Technical Series, Report of Investigations No. 6. Geo-Marine, Inc., Plano.

Lorrain, D. and N. Hoffrichter

1968 Archeological Survey and Excavation at Pat Mayse Reservoir, Texas. Archaeological Salvage Project, Southern Methodist University, Dallas.

Mahoney, R., with contributions by C. Crawford, R. Mauldin, L. Nordt, T. K. Perttula, and S. Reyna

2001 Camp Maxey III, Archaeological Testing of 23 Prehistoric Sites, Lamar County, Texas. Archaeological Survey Report No. 314. Center for Archaeological Research, The University of Texas at San Antonio.

Mallouf, R. J.

1976 Archeological Investigations at Proposed Big Pine Lake, 1974-1975: Lamar and Red River Counties, Texas. Archeological Survey Report No. 18. Office of the State Archeologist, Texas Historical Commission, Austin.

Marceaux, P. S.

2011 The Archaeology and Ethnohistory of the Hasinai Caddo: Material Culture and the Course of European Contact. Ph.D. dissertation, Department of Anthropology, The University of Texas at Austin.

McClurkan, B. B., W. T. Field, and J. N. Woodall

1966 Excavations in Toledo Bend Reservoir, 1964-65. Papers of the Texas Archeological Salvage Project No. 8. Texas Archeological Salvage Project, The University of Texas at Austin. 
McDonald, A. J.

1972 An Archeological Survey of the Martin Lake Area, Rusk and Panola Counties, Texas. Research Report No. 14. Texas Archeological Salvage Project, The University of Texas at Austin.

McGregor, D. E., M. M. Green, D. H. Jurney, W. A. Martin, R. W. Moir, and J. W. Saunders

1996 Archaeological Investigations at Cooper Lake, Delivery Orders Numbers 2, 3 \& 4, 1987. 2 Vols. Archaeology Research Program, Southern Methodist University, Dallas.

Middlebrook, T. A.

1994 An Update of Archaeological Investigations at the Tyson Site. Journal of Northeast Texas Archaeology $3: 1-36$.

Mills, B. J., J. M. Roberts Jr., J. J. Clark, W. R. Haas Jr., D. L. Huntley, M. A. Peeples, L. Borck, S. C. Ryan, M.

A. Trowbridge, and R. L. Breiger

2013 The dynamics of social networks in the late prehispanic U.S. Southwest. In New Approaches in Regional Network Analysis, edited by C. Knappett and R. Rivers, pp. 185-206. Oxford University Press, Oxford.

Miroir, M. E., R. K. Harris, J. C. Blaine, and J. McVay

1973 Bernard de la Harpe and the Nassonite Post. Bulletin of the Texas Archeological Society 44:113-167.

Nash, M. A., S. M. Kotter, and K. V. Reese-Taylor

1995 National Register Testing of Ten Sites in the Monticello B-2 Surface Mine, Titus County, Texas. Document No. 930529. Espey, Huston \& Associates, Inc., Plano.

Nash, M. A., T. K. Perttula, and L. W. Ellis

2012 National Register of Historic Places Eligibility Testing of Site 41 SM404 within TxDOT's Tyler District, Smith County, Texas. Document No. 110055. Atkins, Austin.

Nelson, B. and T. K. Perttula

1993 Site 41UR136, a Titus Phase Site in the Little Cypress Creek Basin. Caddoan Archeology Newsletter 3(4):11-16.

2003a Archeological Investigations of the Underwood Site (41CP230): A Titus Phase Settlement along Big Cypress Creek in Camp County, Texas. Journal of Northeast Texas Archaeology 17:1-61.

2003b Archeological Survey along the Lake Bob Sandlin Shoreline, Camp, Franklin, and Titus Counties, Texas. Report of Investigations No. 46. Archeological \& Environmental Consultants, LLC, Austin.

2006 Archaeological Investigations at the New Hope Site (41FK107) at Lake Bob Sandlin, Franklin County, Texas. Journal of Northeast Texas Archaeology 25:26-37.

Nelson, B., M. Crabb, and T. K. Perttula

2004 The Crabb Site (41TT650), a Prehistoric Caddo Site on Tankersley Creek, Titus County, Texas. Journal of Northeast Texas Archaeology 19:1-21.

Nelson, B., M. Turner, and T. K. Perttula

1996 Archeological Investigations at the Griffin Mound Site: A Middle Caddoan Site in Upshur County, Texas. The Cache, Collected Papers on Texas Archeology 3:49-66. Office of the State Archeologist, Texas Historical Commission, Austin.

Nichols, P., M. Parsons, M. D. Freeman, L. Banks, D. Shanabrook, and B. Rader

1997 Test Excavations at Proposed Lake Gilmer, Upshur County, Texas. Horizon Environmental Services, Inc., Austin.

Parsons, $\mathrm{M}$.

2011 Mitigation Phase Archeological Investigations at Lake Gilmer, Upshur County, Texas. MS on file, Archeology Division, Texas Historical Commission, Austin. 
Parsons, M. L., J. E. Bruseth, J. Bagur, S. E. Goldborer, and C. McCrocklin

2002 Finding Sha'chahdinnih (Timber Hill): The Last Village of the Kadohadacho in the Caddo Homeland. Archeological Reports Series No. 3. Texas Historical Commission, Austin.

Peeples, M. A. and J. M. Roberts Jr.

2013 To binarize or not to binarize: relational data and the construction of archaeological networks. Journal of Archaeological Science 40:3001-3010.

Perttula, T. K.

1998 Caddo Ceramics from the Middle Caddoan Period Knight's Bluff Site (41CS14), Cass County, Texas. Caddoan Archeology 8(4):11-19.

1999 (Editor) The Hurricane Hill Site (41HP106): The Archaeology of a Late Archaic/Early Ceramic and Early-Middle Caddoan Settlement in Northeast Texas. 2 Vols. Special Publication No. 4. Friends of Northeast Texas Archaeology, Pittsburg and Austin.

2000 The Caddoan Ceramics from the Gray's Pasture Site (41HS524), Harrison County, Texas. Journal of Northeast Texas Archaeology 13:1-38.

2002a Caddo Ceramics from 41HS835, Harrison County, Texas. In A Phase I Cultural Resources Survey of the Blocker-Crossroads WSC Water Line Project in Central Harrison County, Texas, by W. E. Moore, pp. AII 1-13. Contract Report No. 103. Brazos Valley Research Associates, Bryan.

2002b (Editor) Archeological Investigations at the Proposed Lake Naconiche, Nacogdoches County, Texas. 2 Vols. Report of Investigations No. 42. Archeological and Environmental Consultants, LLC, Austin.

2005a The M. W. Burks Site (41WD52), A Late Caddo Hamlet in Wood County, Texas. Journal of Northeast Texas Archaeology 23:1-27.

2005b The Cranfill Site (41BW171), a Prehistoric Caddo Site in the Red River Valley, Bowie County, Texas. Journal of Northeast Texas Archaeology 22:1-37.

2005c 1938-1939 WPA Excavations at the Hatchel Site (41BW3) on the Red River in Bowie County, Texas. Southeastern Archaeology 24(2):180-198.

2005d (Editor) Archeological Investigations at the Pilgrim's Pride Site (41CP304), a Titus Phase Community in the Big Cypress Creek Basin, Camp County, Texas. 2 Vols. Report of Investigations No. 30. Archeological \& Environmental Consultants, LLC, Austin.

2008a Analysis of the Historic Caddo Ceramics from 41NA223 in Downtown Nacogdoches, Nacogdoches County, Texas. Journal of Northeast Texas Archaeology 28:35-50.

2008b (Editor) Lake Naconiche Archeology, Nacogdoches County, Texas: Results of the Data Recovery Excavations at Five Prehistoric Archeological Sites. 2 Vols. Report of Investigations No. 60. Archeological \& Environmental Consultants, LLC, Austin.

2008c The Archeology of the Roitsch Site (41RR16), an Early to Historic Caddo Period Village on the Red River in Northeast Texas. In Collected Papers from Past Texas Archeological Society Summer Field Schools, edited by T. K. Perttula, pp. 313-628. Special Publication No. 5. Texas Archeological Society, San Antonio.

2008d Archeological Survey of the Roitsch Farm and Adjoining Lands, 1991 and 1992 Texas Archeological Society Field School, Red River County, Texas. In Collected Papers from Past Texas Archeological Society Summer Field Schools, edited by T. K. Perttula, pp. 173-312. Special Publication No. 5. Texas Archeological Society, San Antonio.

2009a Frankston Phase Ceramics from the Alcoa \# 1 (41AN87) Site, Mound Prairie Creek, Anderson County, Texas. Journal of Northeast Texas Archaeology 29:23-44.

2009b The Ceramics from a Late Caddo Site on Mud Creek in Cherokee County, Texas. Journal of Northeast Texas Archaeology 29:45-52.

2009c Late Caddo Ceramics from 41HE337 in Henderson County, Texas. Journal of Northeast Texas Archaeology 29:53-57. 
2009d Ceramic Vessel Sherds from the Kah-Hah-Ko-Wha Site (41CE354), an Allen Phase Component in Northwestern Cherokee County, Texas. Journal of Northeast Texas Archaeology 29:59-79.

2009e The Archaeology of the 16th and 17th Century Caddo in the Post Oak Savannah of Northeast Texas: The Tuinier Farm (41HP237), R. A. Watkins (41HP238), and Anglin (41HP240) Sites in the Stouts Creek Basin, Hopkins County, Texas. Journal of Northeast Texas Archaeology 30:1-132.

2009f Analysis of the Caddo Archeological Materials from the 1985 Texas Archeological Society Field School at the Washington Square Mound Site, Nacogdoches County, Texas. Bulletin of the Texas Archeological Society 80:145-193.

2009g The Decorated Ceramic Sherds, Plain Rims, and Clay Pipe Sherds from the Stallings Site (41LR297), Lamar County, Texas. MS on file.

2010a Documentation of Caddo Ceramic Vessel Sherds from the Shelby Site (41CP71) in the Vernon Holcomb Collection, Camp County, Texas. Journal of Northeast Texas Archaeology 33:25-29.

$2010 \mathrm{~b}$ Analysis of the Prehistoric Caddo Ceramics from 41LR351, Lamar County, Texas. Journal of Northeast Texas Archaeology 33:69-91.

2010c Analysis of Caddo Sherds from Sites in Shelby County, Texas. Ms on file.

2011a A Radiocarbon Date from a Middle Caddo Period Habitation Site on Hickory Creek, Houston County, Texas. Caddo Archeology Journal 21:147-156.

2011b Another Look at the Grace Creek \#1 Site in Gregg County, Texas, as Seen Through Ceramic Analysis. Journal of Northeast Texas Archaeology 35:11-46.

2011c (assembler) Archaeological and Archaeogeophysical Investigations at an Early Caddo Mound Center in the Sabine River Basin of East Texas, Special Publication No. 15. Friends of Northeast Texas Archaeology, Austin and Pittsburg.

2012 Analysis of Ceramic Sherds from the Mid-18th Century Gilbert Site on Lake Fork Creek, Rains County, Texas. Journal of Northeast Texas Archaeology 37:1-22.

2013a The Sam D. Carpenter Bottom Site (41CP495) in the Big Cypress Creek Basin, Camp County, Texas. Journal of Northeast Texas Archaeology 42:1-21.

2013b The McMinn Ranch Site (41CP72) in the Dry Creek Valley, Camp County, Texas. Journal of Northeast Texas Archaeology 42:23-32.

2013c Analysis of the Prehistoric Artifact Assemblage of Ceramic and Lithic Artifacts from 41LR351, Lamar County, Texas. Journal of Northeast Texas Archaeology 39:7-41.

2013d The Tom Hanks Site (41CP239): A Late Caddo, Titus Phase Mound Site in the Big Cypress Creek Basin, Camp County, Texas. Journal of Northeast Texas Archaeology 41:17-26.

2013e The Linebarger Site on Dry Creek, Camp County, Texas. Journal of Northeast Texas Archaeology 40:3134.

2013f Analysis of Surface Collections from Areas A and B at the Sam Roberts Site (41CP8) on Prairie Creek, Camp County, Texas. Journal of Northeast Texas Archaeology 40:39-46.

2013g The Sam D. Carpenter Garden Plot Site (41CP496), Camp County, Texas. Journal of Northeast Texas Archaeology 40:47-52.

2013h The Analysis of the Aboriginal Ceramic Sherds Recovered from the Keystone Pipeline Project in Eastern Texas. MS on file, SWCA, Inc., Austin.

2013 C Caddo Ceramics in East Texas. Bulletin of the Texas Archeological Society 84:181-212.

2014a Caddo Ceramics from Mound Deposits at the Shelby Mound Site (41CP71) on Greasy Creek, Camp County, Texas. Journal of Northeast Texas Archaeology 46:7-43.

2014b Archaeological Studies of the Hatchel Site (41BW3) on the Red River in Bowie County, Texas. Special Publication No. 23. Friends of Northeast Texas Archaeology, Austin and Pittsburg.

2014c The Mitchell Site (41BW4): An Ancestral Caddo Settlement and Cemetery on McKinney Bayou, Bowie County, Texas. Special Publication No. 32. Friends of Northeast Texas Archaeology, Austin and Pittsburg. 
2014d The Caddo Archaeology of the Musgano Site (41RK19) in the Sabine River Basin of East Texas. Special Publication No. 28. Friends of Northeast Texas Archaeology, Austin and Pittsburg.

2014e The Eli Moores Site, a 17th to early 18th Century Caddo Site on the Red River, Bowie County, Texas. Special Publication No. 31. Friends of Northeast Texas Archaeology, Austin and Pittsburg.

2014f The Hale and Keith Mounds in the Big Cypress Creek Basin in East Texas. Special Publication No. 33. Friends of Northeast Texas Archaeology, Austin and Pittsburg.

2014g Aboriginal Ceramic Vessel and Pipe Sherds from the Murvaul Creek Site (41PN175), Panola County, Texas. MS on file, Versar-Geo-Marine, Inc., Plano.

2014h The Caddo Ceramic and Lithic Assemblage from the Robert Griffin Site (41SY41), Shelby County, Texas. Research Report No. 1. Center for Regional Heritage Research, Stephen F. Austin State University, Nacogdoches.

2014i The Caddo Ceramic Assemblage from the Buddy Hancock Site (41SY45), Shelby County, Texas. Research Report No. 2. Center for Regional Heritage Research, Stephen F. Austin State University, Nacogdoches.

2015a The Caddo Ceramic Assemblage from the Hardin A Site (41GG69) on the Sabine River in Gregg County, Texas. Journal of Northeast Texas Archaeology 51, this volume.

2015b The Harling Site (41FN1), An Ancestral Caddo Mound Site on the Red River in Fannin County, Texas. Journal of Northeast Texas Archaeology 51, this volume.

2015c The Womack Site (41LR1), an Ancestral Caddo Settlement on the Red River in Lamar County, Texas. Journal of Northeast Texas Archaeology 52, in press.

2015d A Titus Phase Midden Mound at the Earl Jones Farm (41WD3) in the Lake Fork Creek Basin, Wood County, Texas. Journal of Northeast Texas Archaeology 53, in press.

2015e The L. L. Winterbauer Site (41WD6), Wood County, Texas. Journal of Northeast Texas Archaeology 53, in press.

$2015 f$ The A. C. Gibson Site (41WD1), a Middle Caddo Period Component on the Sabine River in Wood County, Texas. Journal of Northeast Texas Archaeology 53, in press.

Perttula, T. K. and L. W. Ellis

2012 The Hickory Hill Site (41CP408): Archeological Investigations at a Middle Caddo Site in the Little Cypress Creek Basin in East Texas. Document No. 120055. Atkins Group, Austin.

Perttula, T. K. and K. K. Gilmore

1988 Archaeological Survey along Mill Race Creek and Tributaries, Wood County, Texas: 1987-1988. Contributions in Archaeology No. 6. Institute of Applied Sciences, University of North Texas, Denton.

Perttula, T. K. and T. Middlebrook

2009 Prehistoric Caddo Ceramics from the Henry Lake Site (41CE324), Cherokee County, Texas. Journal of Northeast Texas Archaeology 29:9-21.

Perttula, T. K. and B. Nelson

1997 41HS574, The Coleman Farm Site on Starkey Creek. Journal of Northeast Texas Archaeology 10:52-57.

1998 Archeological Survey Investigations of Selected Parts of the Walker Creek Project Area for Pilgrim's Pride Corporation, Camp County, Texas. Report of Investigations No. 22. Archeological and Environmental Consultants, Austin.

1999 Additional Archeological Survey and Shovel Testing Investigations in the Walker Creek Complex Project Area for Pilgrim's Pride Corporation, Camp County, Texas. Report of Investigations No. 23. Archeological and Environmental Consultants, LLC, Austin.

2000a Phase II Archeological Survey Investigations of the City of Tyler-Lake Palestine WTP Project, Smith County, Texas. Report of Investigations No. 41. Archeological and Environmental Consultants, Austin.

2000b Archeological Investigations at 41CE299, Double Creek Wastewater Treatment Plant, and along Ragsdale Creek, Cherokee County, Texas. Report of Investigations No. 36. Archeological \& Environmental Consultants, LLC, Austin. 
2001 Archeological Test Excavations at the Prestonwood (41SM272) and Broadway (41SM273) Sites along the City of Tyler-Lake Palestine WTP Project, Smith County, Texas. Report of Investigations No. 43. Archeological and Environmental Consultants, LLC, Austin.

2002a An Archeological Survey of Harrison Bayou Lease Lands at the Longhorn Army Ammunition Plant, Harrison County, Texas. Report of Investigations No. 12. Archeological and Environmental Consultants, Austin.

2002b Archeological Survey of Lake Bob Sandlin State Park, Titus County, Texas. Report of Investigations No. 48. Archeological and Environmental Consultants, LLC, Austin.

2003a The Nawi haia ina Site (41RK170): Archeological Investigations in the City of Henderson's Southside Wastewater Treatment Plant, Rusk County, Texas. Report of Investigations No. 51. Archeological \& Environmental Consultants, LLC, Austin.

2003b Archeological Investigations of Village Areas at the Hatchel Site (41BW3), Bowie County, Texas. Report of Investigations No. 58. Archeological \& Environmental Consultants, LLC, Austin.

2004a Archeological Investigations at the Shelby Site (41CP71) on Greasy Creek, Camp County, Texas. Special Publication No. 5. Friends of Northeast Texas Archaeology, Pittsburg and Austin.

2004b Woodland and Caddo Archeology at the Broadway or Kanduts'ah Kuhnihdahahdisa' Site (41SM273) on the City of Tyler-Lake Palestine WTP Project, Smith County, Texas. Report of Investigations No. 50. Archeological \& Environmental Consultants, LLC, Austin.

2006a Archaeological Investigations at the Polk Estates Site (41CP245), Camp County, Texas. Journal of Northeast Texas Archaeology 24:1-83.

2006b Test Excavations at Three Caddo Sites at Mission Tejas State Park, Houston County, Texas. Report of Investigations No. 76. Archeological \& Environmental Consultants, LLC, Austin.

2007a Archeological Survey Investigations and Test Excavations at 41 CE354 at the North and South Lake areas of the H.R.C. Cherokee Tree Farm, L. P. Project, Cherokee County, Texas. Report of Investigations No. 80. Archeological \& Environmental Consultants, LLC, Austin.

2007b Archeological Investigations in 2007 at Mission Tejas State Park in Houston County, Texas. Report of Investigations No. 85. Archeological \& Environmental Consultants, LLC, Austin.

2009 Archeological Survey of 361.3 Acres of the H.R.C. Cherokee Tree Farm, L. P. Project in the Flat Creek Valley, Cherokee County, Texas. Report of Investigations No. 98. Archeological \& Environmental Consultants, LLC, Austin.

2012a The Caddo Ceramic Assemblage from the New Hope Site (41FK107), Franklin County, Texas. Journal of Northeast Texas Archaeology 38:45-71.

2012b The Wa'akas Site (41CP490) at Lake Bob Sandlin, Camp County, Texas. Journal of Northeast Texas Archaeology 38:73-83.

2013 Two Middle Caddo Period Habitation Sites and Cemeteries in the Sabine River Basin, Gregg County, Texas. Special Publication No. 27. Friends of Northeast Texas Archaeology, Pittsburg and Austin.

2014 Additional Artifact Collections from the Gardener Site (41CP55), Camp County, Texas. Journal of Northeast Texas Archaeology 46:73-79.

Perttula, T. K. and R. Z. Selden Jr.

2014 Ceramic Sherds from the Morse Mounds Site (41SY27). Research Report No. 3. Center for Regional Heritage Research, Stephen F. Austin State University, Nacogdoches.

2015 Ancestral Caddo Ceramics in East Texas. Journal of Northeast Texas Archaeology 48:9-58.

Perttula, T. K. and D. L. Sherman

2009 Data Recovery Investigations at the Ear Spool Site (41TT653), Titus County, Texas. Document No. 070205. PBS\&J, Austin.

Perttula, T. K. and B. D. Skiles

2014 The Steck Site (41WD529), a Titus Phase Settlement in the Lake Fork Creek Drainage Basin, Wood County, Texas. Journal of Northeast Texas Archaeology 48:1-8. 
Perttula, T. K. and M. Thacker

2014 Analysis of New Artifact Collections from Archaic to Ancestral Caddo Sites in the Saline Creek Basin in Northern Smith County, Texas. Journal of Northeast Texas Archaeology 43:1-25.

Perttula, T. K. and C. P. Walker

2008 The History of Archaeological Investigations and Geophysical Survey at the Jamestown Mound Site (41SM54), an Archaeological Conservancy Preserve in Smith County, Texas. Archeological \& Environmental Consultants, LLC and Archaeo-Geophysical Associates, LLC, Austin.

Perttula, T. K. and M. Walters

2012 Caddo Sites in the Saline Creek Basin in Northern Smith County, Texas. Journal of Northeast Texas Archaeology 36:47-63.

Perttula, T. K., L. L. Bush, L. Schniebs, T. Middlebrook, and P. S. Marceaux

2010c An Early Historic Caddo Farmstead at the Henry M. Site (41NA60) in Nacogdoches County, Texas. Stephen F. Austin State University Press, Nacogdoches.

Perttula, T. K., D. B. Kelley, and R. A. Ricklis (assemblers and editors)

2011b Archeological Investigations at the Lang Pasture Site (41AN38) in the Upper Neches River Basin of East Texas. Report No. 129. Texas Department of Transportation, Archeological Studies Program, Environmental Affairs Division, Austin.

Perttula, T. K., B. Nelson, and P. Haskins

2012c Additional Lake Bob Sandlin Sites with Documented Collections of Prehistoric Lithic and Ceramic Artifacts. Journal of Northeast Texas Archaeology 38:35-44.

Perttula, T. K., B. Nelson, and R. Z. Selden, Jr.

2014a The Gardener Site (41CP55): A Late Caddo Settlement on Big Cypress Creek in East Texas. Journal of Northeast Texas Archaeology 44:1-11.

Perttula, T. K., B. Nelson, and M. Walters

2004 The South Lilly \#4 Site (41UR279), Upshur County, Texas. Journal of Northeast Texas Archaeology 19:22-60.

2011a Archaeological Sites Along King Creek in Western Nacogdoches County, in East Texas. Journal of Northeast Texas Archaeology 34:69-77.

2011c Archeological Survey Investigations to Identify 17th-early 19th Century Caddo Sites along El Camino Real de los Tejas National Historic Trail in East Texas. Report of Investigations No. 108. Archeological \& Environmental Consultants, LLC, Austin.

2012a The Buckner Dam Site (41CE339) and Four Other Caddo Sites on Gum Creek in the Upper Neches River Basin, Cherokee County, Texas. Journal of Northeast Texas Archaeology 36:65-75.

2013b Archaeological Investigations at the Pine Creek Site, an Allen Phase Settlement on Flat Creek in Northwestern Cherokee County, Texas. Journal of Northeast Texas Archaeology 40:1-18.

2014b Renewed Archaeological Investigations at the Sanders Site (41LR2), Lamar County, Texas. Journal of Northeast Texas Archaeology 47:25-30.

Perttula, T. K., B. Nelson, M. Walters, and L. Schniebs

2007 Archaeological Investigations of the Lang Pasture (41AN38) Midden Deposits on private property west of the SH 155 Right-of-Way, Anderson County, Texas. Caddo Archeology Journal 16:27-36.

Perttula, T. K., B. Nelson, M. Walters, and R. Z. Selden Jr.

2015 The Sanders Site (41LR2): A Middle to Historic Caddo Settlement and Mound Center on the Red River in Lamar County, Texas. Journal of Northeast Texas Archaeology 50, in press.

Perttula, T. K., R. Z. Selden, Jr., and B. Nelson

2013a Analysis of the Ceramic Sherds from Area C at the Ware Acres Site (41GG31), Gregg County, Texas. Journal of Northeast Texas Archaeology 41:57-79. 
Perttula, T. K., B. D. Skiles, and B. C. Yates

1993a The Carlisle Site (41WD46), a Middle Caddoan Occupation on the Sabine River, Wood County, Texas. Notes on Northeast Texas Archaeology 1:34-62.

1993b The Goldsmith Site (41WD208): Investigations of the Titus Phase in the Upper Sabine River Basin, Northeast Texas. Bulletin of the Texas Archeological Society 61:139-191.

Perttula, T. K., M. B. Trubitt, and J. S. Girard

2012e The Use of Shell-Tempered Pottery in the Caddo Area of the Southeastern United States. Southeastern Archaeology 30(2):242-267.

Perttula, T. K., M. Walters, and B. Nelson

2010a Documenting Caddo Ceramic Sherd and Lithic Collections from Prehistoric Sites at Lake Bob Sandlin. Journal of Northeast Texas Archaeology 33:31-39.

2010b Further Investigations of a Prehistoric Caddo Habitation Site in the White Oak Creek Basin of Northeast Texas: The James Owens Site (41TT769). Caddo Archeology Journal 20:53-76.

2012b The Younger Site (41MR6), Marion County, Texas. Journal of Northeast Texas Archaeology 38:1-20.

2012d Archeological Investigations at the Pace McDonald Site (41AN51): A Middle Caddo Mound Center in the Neches River Basin in East Texas. Special Publication No. 21. Friends of Northeast Texas Archaeology, Pittsburg and Austin.

Perttula, T. K., B. Young, and P. S. Marceaux

2009 Caddo Ceramics from an Early $18^{\text {th }}$ Century Spanish Mission in East Texas: Mission San Jose de los Nasonis (41RK200). Journal of Northeast Texas Archaeology 29:81-89.

Prikryl, D. J.

2008 The 1991 and 1992 Texas Archeological Society Field School Excavations at the Fasken Site (41RR14), Red River County, Texas. In Collected Papers from Past Texas Archeological Society Summer Field Schools, edited by T. K. Perttula,pp. 125-171. Special Publication No. 5. Texas Archeological Society, San Antonio.

Robinson, D. G.

1997 Cultural Resource Investigations at the Ducks Unlimited Marsh (DUM) Project, North Toledo Bend Reservoir, Shelby County, Texas. Report 97-3. Cultural Resource Program, Texas Parks and Wildlife Department, Austin.

Rogers, R. and T. K. Perttula

2004 The Oak Hill Village (41RK214), Rusk County, Texas. Document No. 030083. PBS\&J, Austin.

Rogers, R., M. B. Cliff, T. K. Perttula, G. Rutenberg, S. Victor, P. Dering, and M. Malainey

2003 Excavations at the Alex Justiss Site, 41TT13, Titus County, Texas. Report No. 36. Archeological Studies Program, Texas Department of Transportation, Austin.

Rogers, R., E. Foster, K. Reese-Taylor, G. Rutenberg, M. Nash, J. Hageman, and D. Jurney

1994 National Register Testing at Eight Archaeological Sites within the Oak Hill 2,280-Acre Study Area, Rusk County, Texas. Document No. 930169. Espey, Huston \& Associates, Inc., Austin.

Scurlock, J. D.

1962 The Culpepper Site, a Late Fulton Aspect Site in Northeast Texas. Bulletin of the Texas Archeological Society 32:285-316.

Selden, R. Z. Jr. and T. K. Perttula

2014 Archaeological Sites on the Sabine National Forest, Sabine and Shelby Counties, Texas. Research Report No. 7. Center for Regional Heritage Research, Stephen F. Austin State University, Nacogdoches.

Selden, R. Z. Jr., T. K. Perttula, and D. L. Carlson

2014 INAA and the provenance of shell-tempered sherds in the ancestral Caddo region. Journal of Archaeological Science 47:113-120. 
Shafer, H. J.

1981 Archeological Investigations at the Attaway Site, Henderson County, Texas. Bulletin of the Texas Archeological Society 52:147-179.

Sherman, D. L.

2001 NRHP Eligibility Testing (41RK107, 41RK240, 41RK242, 41RK243, 41RK276, and 41RK286) and Additional Testing (41RK243) Investigations within the Oak Hill DIII Mine, Permit No. 46, Rusk County, Texas. Document No. 000237. PBS\&J, Austin.

2004 National Register Testing of Site 41CP408: A Middle Caddoan Farmstead, Camp County, Texas. Document No. 040031. PBS\&J, Austin.

Skinner, S. A., R. K. Harris, and K. M. Anderson (editors)

1969 Archaeological Investigations at the Sam Kaufman Site, Red River County, Texas. Contributions in Anthropology No. 5. Department of Anthropology, Southern Methodist University, Dallas.

Smith, N. G., A. Karasik, T. Narayanan, E. S. Olson, U. Smilanksy, and T. E. Levy

2014 The Pottery Informatics Query Database: A New Method for Mathematic and Quantitative Analyses of Large Regional Ceramic Datasets. Journal of Archaeological Method and Theory 21(1):212-250.

Stokes, J. and J. Woodring

1981 Native-Made Artifacts of Clay. In Archeological Investigations at the George C. Davis Site, Cherokee County, Texas: Summers of 1979 and 1980, edited by D. A. Story, pp. 135-238. Occasional Paper No. 1. Texas Archeological Research Laboratory, The University of Texas at Austin.

Story, D. A.

1965 The Archeology of Cedar Creek Reservoir, Henderson and Kaufman Counties, Texas. Bulletin of the Texas Archeological Society 36:163-257.

Story, D. A., B. Barber, E. Cobb, H. Cobb, R. Coleman, K. Gilmore, R. K. Harris, and N. Hoffrichter

1967 Pottery Vessels. In "The Gilbert Site: A Norteno Focus Site in Northeast Texas," edited by E. B. Jelks. Bulletin of the Texas Archeological Society 37:112-187.

Suhm, D. A. and E. B. Jelks (editors)

1962 Handbook of Texas Archeology: Type Descriptions. Special Publication No. 1, Texas Archeological Society, and Bulletin No. 4, Texas Memorial Museum, Austin. Reprinted in 2009, Gustav's Library, Davenport, Iowa.

Sundermeyer, S. A., J. T. Penman, and T. K. Perttula

2008 Integrated Cultural Resources Investigations for the Bowie County Levee Realignment Project, Bowie County, Texas, and Little River County, Arkansas. Miscellaneous Reports, Report of Investigations No. 29. LopezGarcia Group, Dallas.

Thurmond, J. P.

1990 Archeology of the Cypress Creek Drainage Basin, Northeastern Texas and Northwestern Louisiana. Studies in Archeology 5. Texas Archeological Research Laboratory, The University of Texas at Austin.

Thurmond, J. P. and U. Kleinschmidt

1979 Report on the Fall 1978 Investigations at the George C. Davis Site, Caddoan Mounds State Historic Site, Cherokee County, Texas. Texas Archeological Research Laboratory, The University of Texas at Austin.

Turner, R. L. and J. E. Smith II

2002 The Harold Williams Site (41CP10) and the Texas Archeological Society Field School of 1967. Bulletin of the Texas Archeological Society 73:1-68.

Walker, C. P. and T. K. Perttula

2011 Archaeogeophysics and Archaeological Investigations at a Historic Caddo Site Along El Camino Real de los Tejas: The J.T. King Site (41NA15) in Nacogdoches County, Texas. Archaeo-Geophysical Associates, LLC and Archeological \& Environmental Consultants, LLC, Austin. 
Walters, $\mathrm{M}$.

2003 The Wolf Site (41SM195), Smith County, Texas. Journal of Northeast Texas Archaeology 18:1-21.

2006 The Lake Clear (41SM243) Site and Crotalus horridus atricaudatus. Caddoan Archeology Journal 15:5-41.

2009 The Henry Chapman Site (41SM56). Journal of Northeast Texas Archaeology 31:11-35.

Walters, M., with contributions from L. G. Cecil, L. S. Cummings, J. P. Dering, J. R. Ferguson, M. D. Glascock,

T. K. Perttula, L. Schniebs, H. J. Shafer, J. Todd, and C. P. Walker

2008 Life on Jackson Creek, Smith County, Texas: Archeological Investigations of a 14th Century Caddo Domicile at the Leaning Rock Site (41SM325). Caddo Archeology Journal 17:1-114.

Walters, M. and P. Haskins

1998 Archaeological Investigations at the Redwine Site (41SM193), Smith County, Texas. Journal of Northeast Texas Archaeology 11:1-38.

2000 The Bryan Hardy Site (41SM55), Smith County, Texas. Journal of Northeast Texas Archaeology 12:1-26.

Walters, M. and T. K. Perttula

2012 Certain Caddo Sites on Stone Chimney Creek, Cherokee County, Texas. Journal of Northeast Texas Archaeology 37:37-88.

Walters, M., B. Boyd, B. Nelson, T. K. Perttula, and L. Schniebs

2003 The James Owens Site (41TT769) in the Sulphur River Basin of Northeast Texas. Caddoan Archeology Journal 13(1):16-34.

Webb, C. H., F. E. Murphey, W. G. Ellis, and H. R. Green

1969 The Resch Site, 41HS16, Harrison County, Texas. Bulletin of the Texas Archeological Society 40:3-106.

Woodall, J. N.

1969 Archeological Excavations in the Toledo Bend Reservoir, 1966. Contributions in Anthropology No. 3. Department of Anthropology, Southern Methodist University, Dallas.

Wormser, A. J.

1991 Test Excavations at the Jodie Bender Site, 41HS11, Harrison County, Texas. Texas State Department of Highways and Public Transportation, Highway Design Division, Austin. 